TUW-04-12

\title{
Non-Fermi-Liquid Specific Heat of Normal Degenerate Quark Matter
}

\author{
A. Gerhold, A. Ipp, and A. Rebhan \\ Institut für Theoretische Physik, Technische Universität Wien, \\ Wiedner Haupstr. 8-10, A-1040 Vienna, Austria
}

(Dated: November 1, 2018)

\begin{abstract}
We compute the low-temperature behavior of the specific heat of normal (non-colorsuperconducting) degenerate quark matter as well as that of an ultradegenerate electron gas. Long-range magnetic interactions lead to non-Fermi-liquid behavior with an anomalous leading $T \ln T^{-1}$ term. Depending on the thermodynamic potential used as starting point, this effect appears as a consequence of the logarithmic singularity in the fermion self-energy at the Fermi surface or directly as a contribution from the only weakly screened quasistatic magnetic gauge bosons. We show that a calculation of Boyanovsky and de Vega claiming the absence of a leading $T \ln T^{-1}$ term missed it by omitting vector boson contributions to the internal energy. Using a formulation which collects all nonanalytic contributions in bosonic ring diagrams, we systematically calculate corrections beyond the well-known leading-log approximation. The higher-order terms of the low-temperature expansion turn out to also involve fractional powers $T^{(3+2 n) / 3}$ and we explicitly determine their coefficients up to and including order $T^{7 / 3}$ as well as the subsequent logarithmically enhanced term $T^{3} \ln (c / T)$. We derive also a hard-dense-loop resummed expression which contains the infinite series of anomalous terms to leading order in the coupling and which we evaluate numerically. At low temperatures, the resulting deviation of the specific heat from its value in naive perturbation theory is significant in the case of strongly coupled normal quark matter and thus of potential relevance for the cooling rates of (proto-)neutron stars with a quark matter component.

PACS numbers: 11.10.Wx, 12.38.Mh, 71.45.Gm, 11.15.Pg
\end{abstract}

\section{INTRODUCTION}

It is well-known that long-range magnetic interactions in a degenerate electron gas lead to non-Fermi-liquid behavior which manifests itself in the appearance of an anomalous contribution to the low-temperature limit of entropy and specific heat proportional to $\alpha T \ln T^{-1}$ as discovered by Holstein, Norton, and Pincus [1] over thirty years ago. While this effect is perhaps too small for experimental detection in nonrelativistic situations, it drew renewed theoretical attention more recently [2, 3, 4] after the detection of non-Fermi-liquid behavior in the normal state of hightemperature superconductors [5] and in other systems of strongly correlated electrons, which may be due to effective gauge field dynamics (see also [6, 7, 8]).

In deconfined degenerate quark matter, the analogous effect can more easily be important because the larger coupling constant $\alpha_{s}$ together with the relatively large number of gauge bosons increases the numerical value of the effect by orders of magnitude. In contrast to the case of a hightemperature quark-gluon plasma, chromomagnetostatic fields are expected to remain unscreened in the low-temperature limit [9] and thus lead to the same singularities in the fermion self-energy that are responsible for the breakdown of the Fermi-liquid description in the nonrelativistic electron gas considered in [1].

An important consequence of such non-Fermi-liquid behavior in quantum chromodynamics (QCD) is a reduction of the magnitude of the gap in color superconductors 9, 10, 11] which on the basis of weak-coupling calculations are estimated to have a critical temperature in the range between 6 and $60 \mathrm{MeV}$ [12]. Quark matter above this temperature, and unpaired quark matter component also below it, has long-range chromomagnetic interactions that should lead to an anomalous specific heat with possible relevance for the cooling of young neutron stars as 
pointed out by Boyanovsky and de Vega [13, 14]. However, in Ref. [14] these authors claimed that the $\alpha T \ln T^{-1}$ term in the specific heat as reported in [1, 2, 3] would not exist, neither in QCD nor in QED. Instead they obtained a $\alpha T^{3} \ln T$ correction to the leading ideal-gas behavior, which by renormalization-group arguments was resummed into a $T^{3+O(\alpha)}$ correction as the leading non-Fermi-liquid effect on the specific heat. ${ }^{1}$ At low temperatures, such a contribution would be rather negligible compared to standard perturbative corrections to the ideal-gas result $\propto T$.

In a numerical study of the exactly solvable large-flavor-number limit of QCD and QED [15] at nonzero chemical potential [16], two of us however found that the entropy at low temperature has a behavior suggestive of a $\alpha T \ln T^{-1}$ term. In Ref. [17], the three of us have recently reproduced the known $\alpha T \ln T^{-1}$ term in entropy and specific heat, together with further anomalous higherorder corrections, in an analytical calculation that should apply equally to the case of finite flavor number. This calculation is however organized in a form which does not allow one to compare directly with the calculation of Ref. [14] where all $\alpha T \ln T^{-1}$ terms appeared to cancel.

In this paper we shall therefore investigate the approach of Ref. [14], which derived the specific heat from a formula for the internal energy, and compare with two somewhat more direct calculations, one using a self-consistent formula for the entropy and another using an expression for the thermodynamic potential that becomes exact in the limit of large flavor number.

As we shall demonstrate, all these approaches agree eventually and do give a leading $\alpha T \ln T^{-1}$ term for the specific heat. In the calculation using a self-consistent formula for the entropy (Sect. III) the $\alpha T \ln T^{-1}$ term arises as a contribution from the spectral density of the fermions with their logarithmic singularity in the self-energy. There are also $\alpha T \ln T^{-1}$ contributions from the gauge boson sector, but these cancel in the end, which thus validates the (in our opinion not unquestionable) starting point of the original calculation by Holstein et al. [1]. On the other hand, in the calculation of the specific heat from the internal energy (Sect. III) we find that keeping only the fermionic contributions leads to a cancellation of the leading $\alpha T \ln T^{-1}$ term, just as observed in Ref. 14]. However, it turns out that in this approach the contribution of the gauge bosons to the specific heat cannot be neglected, but now contains the complete leading logarithm.

In Sect. IV we describe the details of a calculation which allows us to systematically go beyond the leading-log approximation. Besides completing the argument of the leading logarithm, we find fractional powers $T^{(3+2 n) / 3}$ and we determine their coefficients up to and including order $T^{7 / 3}$ as well as the subsequent logarithmically enhanced term $T^{3} \ln (c / T)$. This low-temperature expansion requires that the temperature is much smaller than the scale set by the Debye mass. At temperatures of the order of the Debye mass or larger, but still much smaller than the quark chemical potential, a complete leading-order result which contains the infinite series of anomalous terms is obtained in Sect. V. It involves a hard-dense-loop resummed one-loop expression, which we evaluate numerically in Sect. VI This allows us to study the quality of the low-temperature expansion, and to compare with the exact results for the large-flavor-number limit. For the sake of this comparison we shall throughout use the notation

$$
g_{\mathrm{eff}}^{2}=\left\{\begin{array}{l}
\frac{g^{2} N_{f}}{2}, \text { QCD }, \\
g^{2} N_{f}, \text { QED } .
\end{array}\right.
$$

with $g$ the coupling constant and $N_{f}$ the number of quark (or electron) flavors. At finite $N_{f}$ we finally evaluate our results numerically for a range of coupling which may be relevant for (normal) quark matter in (proto-) neutron stars, with the finding that there is an interesting range of temperature where the anomalous specific heat exceeds significantly the ideal-gas value.

\footnotetext{
${ }^{1}$ Resummation of the $\alpha T \ln T^{-1}$ term along the lines of Ref. [14] would have led to a $T^{1+O(\alpha)}$ term instead.
} 


\section{ANOMALOUS SPECIFIC HEAT FROM THE ENTROPY}

The specific heat $\mathcal{C}_{v}$ per unit volume is defined as the logarithmic derivative of the entropy density with respect to temperature at constant volume and number density

$$
\mathcal{C}_{v}=T\left(\frac{\partial \mathcal{S}}{\partial T}\right)_{\mathcal{N}}
$$

This is related to derivatives of the thermodynamic potential with respect to $T$ and $\mu$ by 18 ]

$$
C_{v}=T\left\{\left(\frac{\partial \mathcal{S}}{\partial T}\right)_{\mu}-\left(\frac{\partial \mathcal{N}}{\partial T}\right)_{\mu}^{2}\left(\frac{\partial \mathcal{N}}{\partial \mu}\right)_{T}^{-1}\right\}
$$

but at low temperatures one has

$$
\mathcal{C}_{v}=T\left(\frac{\partial \mathcal{S}}{\partial T}\right)_{\mu}+\mathcal{O}\left(T^{3}\right),
$$

so that both $\mathcal{C}_{v}$ and $\mathcal{S}$ contain the same $T \ln T$ term, if any.

The entropy as first derivative of the thermodynamic potential $\Omega$ with respect to $T$ is in some important respects a simpler quantity than $\Omega$. In gauge theories with fermions the latter is given by the following functional of the full propagators $D$ (for gauge bosons), $S$ (for fermions) 19],

$$
\beta \Omega[D, S]=\frac{1}{2} \operatorname{Tr} \ln D^{-1}-\frac{1}{2} \operatorname{Tr} \Pi D-\operatorname{Tr} \ln S^{-1}+\operatorname{Tr} \Sigma S+\Phi[D, S],
$$

where $\Phi$ is a series of 2-particle-irreducible (skeleton) diagrams and where for simplicity we assumed a ghost-free gauge.

Using the fact that $\Omega[D, S]$ is stationary with respect to variations of $D$ and $S$, one can derive an expression for the entropy which to two-loop order in the skeleton expansion is entirely given by propagators and self-energies [20, 21]. Neglecting the longitudinal gluon mode, and the antiparticle contributions in the fermionic sector, this reads

$$
\begin{aligned}
\mathcal{S}=\left(\frac{\partial P}{\partial T}\right)_{\mu} \simeq- & \int \frac{d^{4} K}{(2 \pi)^{4}}\left[2 N_{g} \frac{\partial n_{b}(\omega)}{\partial T}\left(\operatorname{Im} \ln D_{T}^{-1}-\operatorname{Im} \Pi_{T} \operatorname{Re} D_{T}\right)\right. \\
& \left.+4 N N_{f} \frac{\partial n_{f}(\omega)}{\partial T}\left(\operatorname{Im} \ln S_{+}^{-1}+\operatorname{Im} \Sigma_{+} \operatorname{Re} S_{+}\right)\right]+\mathcal{S}^{\prime},
\end{aligned}
$$

where $D_{T}^{-1}=-\omega^{2}+k^{2}+\Pi_{T}, S_{+}^{-1}=-\omega+k-\Sigma_{+}, n_{b}(\omega)=\left(e^{\omega / T}-1\right)^{-1}$, and $n_{f}(\omega)=\left(e^{(\omega-\mu) / T}+\right.$ $1)^{-1}$.

In the original derivation of the anomalous specific heat in QED by Holstein et al. 1], only the term involving $\operatorname{Im} \ln S_{+}^{-1}$ in the quark part had been taken into account, by way of reference to a formula by Luttinger 22] (Eq. (46) therein), which is fully justified actually only for standard Fermi-liquid systems.

Since the calculation of Ref. 1] has been questioned by Boyanovsky and de Vega [14], we consider the more general expression above. For the moment we are interested only in terms of order $g^{2} T \ln T$, so we can neglect $\mathcal{S}^{\prime}$, which vanishes at two-loop order in the skeleton expansion 20] and should therefore only give contributions which are suppressed by an additional factor of $g^{2}$. 


\section{A. Quark part}

In Eq. (6) we have the following contribution from the quarks,

$$
\begin{aligned}
\mathcal{S}_{(\mathrm{q})}=- & 4 N_{f} \int \frac{d^{4} K}{(2 \pi)^{4}} \frac{\partial n_{f}(\omega)}{\partial T} \\
& \times\left[\operatorname{Im} \ln \left(-\omega+k-\Sigma_{+}\right)+\operatorname{Im} \Sigma_{+} \operatorname{Re} \frac{1}{-\omega+k-\Sigma_{+}}\right] \\
\simeq- & \frac{1}{\pi^{3}} N N_{f} \int_{0}^{\infty} d k k^{2} \int_{-\infty}^{\infty} d \omega \frac{\partial n_{f}(\omega)}{\partial T} \\
& \times\left[\operatorname{Im} \ln (-\omega+k)-\operatorname{Re} \Sigma_{+} \operatorname{Im} \frac{1}{-\omega+k}\right]
\end{aligned}
$$

where we have performed an expansion with respect to $\Sigma_{+}$, keeping only the free term and the term corresponding to a single quark self energy insertion ${ }^{2}$. The free term gives the particle contribution to the free fermionic entropy density,

$$
\mathcal{S}_{(\mathrm{q})}^{\text {free }} \simeq N N_{f} \frac{\mu^{2} T}{3}
$$

In the last term in Eq. (17) the factor $\operatorname{Im} 1 /(-\omega+k)$ forces the self energy to be on the mass shell. Using the expression for $\Sigma_{+}$given in [10, 23],

$$
\Sigma_{+} \simeq \frac{g^{2} C_{f}}{24 \pi^{2}}(\omega-\mu) \ln \left(\frac{M^{2}}{(\omega-\mu)^{2}}\right)+i \frac{g^{2} C_{f}}{12 \pi}|\omega-\mu|,
$$

which is nonanalytic in $\omega$ (but not with respect to $k[10]$ ), we obtain

$$
\mathcal{S}_{(\mathrm{q})}^{N L O}=\frac{N_{g}}{\pi^{2}} \int_{0}^{\infty} d k k^{2} \frac{\partial n_{f}(k)}{\partial T} \frac{g_{\mathrm{eff}}^{2}}{24 \pi^{2}}(k-\mu) \ln \frac{M^{2}}{(k-\mu)^{2}} .
$$

With the substitution $k=T z+\mu$ we find that the integral is dominated by small values of $z$, and therefore we may send the lower integration limit to $-\infty$. Then we obtain at order $T \ln T$

$$
\mathcal{S}_{(\mathrm{q})}^{N L O}=\frac{g_{\mathrm{eff}}^{2} N_{g} \mu^{2} T}{36 \pi^{2}} \ln \left(\frac{M}{T}\right)
$$

This result agrees with the one of Holstein et al. 11] after correcting a factor of 4 therein, as done previously in Ref. [3].

\section{B. Gluon part}

The gluon part $\mathcal{S}_{(\mathrm{g})}$ is given by the first line of Eq. ([6). Using the relation

$$
\operatorname{Im} \ln D^{-1}=\arctan \left(\frac{\operatorname{Im} \Pi}{\operatorname{Re} D^{-1}}\right)-\pi \epsilon(\omega) \theta\left(-\operatorname{Re} D^{-1}\right),
$$

\footnotetext{
${ }^{2}$ Note that, diagrammatically, the part with a single self energy insertion corresponds to the gluon ring diagram of section IIB
} 
we write $\mathcal{S}_{(\mathrm{g})}=\mathcal{S}_{(\text {cut })}+\mathcal{S}_{(\Pi)}+\mathcal{S}_{(\text {pole })}$, with

$$
\begin{aligned}
& \mathcal{S}_{(\mathrm{cut})}=2 N_{g} \int \frac{d^{4} K}{(2 \pi)^{4}} \frac{\partial n_{b}(\omega)}{\partial T} \arctan \left(\frac{\operatorname{Im} \Pi_{T}}{\omega^{2}-k^{2}-\operatorname{Re} \Pi_{T}}\right), \\
& \mathcal{S}_{(\Pi)}=-2 N_{g} \int \frac{d^{4} K}{(2 \pi)^{4}} \frac{\partial n_{b}(\omega)}{\partial T} \operatorname{Im} \Pi_{T} \operatorname{Re} \frac{1}{\omega^{2}-k^{2}-\Pi_{T}}, \\
& \mathcal{S}_{(\text {pole })}=2 N_{g} \int \frac{d^{4} K}{(2 \pi)^{4}} \frac{\partial n_{b}(\omega)}{\partial T} \pi \epsilon(\omega) \theta\left(\omega^{2}-k^{2}-\operatorname{Re} \Pi_{T}\right)
\end{aligned}
$$

where we again neglect the contribution of the Debye-screened longitudinal gluons. For the cut term we use the approximation $\omega \ll k$, because it can be checked that including terms of higher order in $\omega$ would only produce terms of higher order than $T \ln T$ (see section IV). In this region we have

$$
\Pi_{T} \simeq-i \frac{g_{\mathrm{eff}}^{2} \mu^{2} \omega}{4 \pi k}
$$

Introducing an UV-cutoff $k_{c}$ for the moment, we obtain

$$
\mathcal{S}_{(\mathrm{cut})} \simeq \frac{N_{g}}{2 \pi^{3}} \int_{0}^{k_{c}} d k k^{2} \int_{-\infty}^{\infty} d \omega \frac{\partial n_{b}(\omega)}{\partial T} \arctan \left(\frac{g_{\mathrm{eff}}^{2} \mu^{2} \omega}{4 \pi k^{3}}\right) .
$$

In order to evaluate this integral we make the substitution $y=\omega / T, x=4 \pi k^{3} /\left(g_{\mathrm{eff}}^{2} \mu^{2} T\right)$. Keeping only the term of order $T \ln T$, we obtain the cutoff-independent result

$$
\mathcal{S}_{(\mathrm{cut})} \simeq \frac{g_{\mathrm{eff}}^{2} N_{g} \mu^{2} T}{36 \pi^{2}} \ln \left(\frac{M^{\prime}}{T}\right) .
$$

The determination of the constant $M^{\prime}$ requires a more accurate calculation and will be carried out in Sect. IV

Next we evaluate $\mathcal{S}_{(\Pi)}$. Following similar steps as in the computation of $\mathcal{S}_{(\text {cut })}$, we find

$$
\begin{aligned}
\mathcal{S}_{(\Pi)} & \simeq 2 N_{g} \int_{0}^{k_{c}} \frac{d k k^{2}}{2 \pi^{2}} \int_{-\infty}^{\infty} \frac{d \omega}{2 \pi} \frac{\partial n_{b}(\omega)}{\partial T}\left(-\frac{g_{\mathrm{eff}}^{2} \mu^{2} \omega}{4 \pi k}\right) \frac{k^{2}}{k^{4}+\left(\frac{g_{\mathrm{eff}}^{2} \mu^{2} \omega}{4 \pi k}\right)^{2}} \\
& \simeq-\frac{g_{\mathrm{eff}}^{2} N_{g} \mu^{2} T}{36 \pi^{2}} \ln \left(\frac{M^{\prime}}{T}\right) .
\end{aligned}
$$

We observe that at order $T \ln T$ this expression just cancels the contribution from Eq. (18).

Finally we consider the pole part. To leading order, i.e. in the hard dense loop (HDL) approximation 24, 25, 26, 27], we have at low temperature $\mu \partial \Pi_{T} / \partial \mu \simeq 2 \Pi_{T}$, and therefore

$$
\begin{aligned}
\mu \frac{\partial \mathcal{S}_{(\text {pole })}}{\partial \mu} & =-2 N_{g} \int \frac{d^{4} K}{(2 \pi)^{4}} \frac{\partial n_{b}(\omega)}{\partial T} \pi \epsilon(\omega) \delta\left(\omega^{2}-k^{2}-\operatorname{Re} \Pi_{T}\right) 2 \operatorname{Re} \Pi_{T} \\
& =-4 \pi N_{g} \int \frac{d^{4} K}{(2 \pi)^{4}} \frac{\partial n_{b}(\omega)}{\partial T}\left(\omega^{2}-k^{2}\right) \epsilon(\omega) \delta\left(\omega^{2}-k^{2}-\operatorname{Re} \Pi_{T}\right),
\end{aligned}
$$

where we have discarded contributions $\sim T^{3}$ which are negligible in the low-temperature limit. Using [28]

$$
\epsilon(\omega) \delta\left(\operatorname{Re} D_{T}^{-1}\right)=Z_{T}(k)\left[\delta\left(\omega-\omega_{T}(k)\right)-\delta\left(\omega+\omega_{T}(k)\right)\right]
$$


we find

$$
\mu \frac{\partial \mathcal{S}_{(\text {pole })}}{\partial \mu}=-\frac{2 N_{g}}{\pi^{2}} \int_{0}^{\infty} d k k^{2} \frac{\partial n_{b}\left(\omega_{T}(k)\right)}{\partial T}\left(\omega_{T}(k)^{2}-k^{2}\right) Z_{T}(k)
$$

We can estimate this integral as follows. Assuming $T \ll \omega_{p} \propto g_{\text {eff }} \mu$, we have the inequalities

$$
\begin{aligned}
\int_{0}^{\infty} & d k k^{4} \frac{\partial n_{b}\left(\omega_{T}(k)\right)}{\partial T} Z_{T}(k)<\int_{0}^{\infty} d k k^{4} \frac{\partial n_{b}\left(\omega_{T}(k)\right)}{\partial T} \frac{1}{2 k} \\
< & \frac{1}{2} \int_{0}^{\omega_{p}} d k k^{3} \frac{\partial n_{b}\left(\omega_{p}\right)}{\partial T}+\frac{1}{2} \int_{\omega_{p}}^{\infty} d k k^{3} \frac{\partial n_{b}(k)}{\partial T} \simeq \frac{\omega_{p}^{5}}{8 T^{2}} e^{-\omega_{p} / T}
\end{aligned}
$$

and

$$
\begin{aligned}
\int_{0}^{\infty} & d k k^{2} \frac{\partial n_{b}\left(\omega_{T}(k)\right)}{\partial T} \omega_{T}(k)^{2} Z_{T}(k)<\int_{0}^{\infty} d k k^{2} \frac{\partial n_{b}\left(\omega_{T}(k)\right)}{\partial T}\left(\omega_{p}+k\right)^{2} \frac{1}{2 k} \\
& <\frac{1}{2} \int_{0}^{\omega_{p}} d k k\left(\omega_{p}+k\right)^{2} \frac{\partial n_{b}\left(\omega_{p}\right)}{\partial T}+\frac{1}{2} \int_{\omega_{p}}^{\infty} d k k\left(\omega_{p}+k\right)^{2} \frac{\partial n_{b}(k)}{\partial T} \\
& \simeq \frac{17 \omega_{p}^{5}}{24 T^{2}} e^{-\omega_{p} / T} .
\end{aligned}
$$

Apart from terms $\sim T^{3}$ which are dropped in the derivative with respect to $\mu$, this crude estimate (which we shall refine in Sect. $\nabla$ below) shows that the pole contribution is exponentially suppressed, essentially because of $\omega_{T} \geq \omega_{p}$.

\section{Result}

In total we find the following result for the entropy at low temperature,

$$
\mathcal{S}=\mathcal{S}_{(\mathrm{g})}+\mathcal{S}_{(\mathrm{q})} \simeq N N_{f} \frac{\mu^{2} T}{3}+\frac{g_{\text {eff }}^{2} N_{g} \mu^{2} T}{36 \pi^{2}} \ln \left(\frac{M}{T}\right) .
$$

From Eq. (18) we see that the $T \ln T$ term can also be obtained by starting only from the expression

$$
\mathcal{S} \simeq N N_{f} \frac{\mu^{2} T}{3}-2 N_{g} \int \frac{d^{4} K}{(2 \pi)^{4}} \frac{\partial n_{b}(\omega)}{\partial T} \operatorname{Im} \ln D_{T}^{-1},
$$

with $D$ the resummed gluon propagator. This formula corresponds to integrating out the fermions, as has indeed been done in the approach of Ref. [2].

On the other hand, we see from Eqs. (17) and (11) that one equally gets the correct result by using only the purely fermionic expression

$$
\mathcal{S} \simeq-4 N N_{f} \int \frac{d^{4} K}{(2 \pi)^{4}} \frac{\partial n_{f}(\omega)}{\partial T} \operatorname{Im} \ln \left(-\omega+k-\operatorname{Re} \Sigma_{+}\right),
$$

which justifies the starting point of Refs. [1, 3]. 


\section{SPECIFIC HEAT FROM THE ENERGY DENSITY}

In Ref. [14], which did not find a term $g^{2} T \ln T^{-1}$ in the specific heat, the starting point was instead the internal energy density.

The energy density can be obtained from the expectation value of the energy momentum tensor,

$$
\mathcal{U}=\frac{1}{V} \int d^{3} x\left\langle T^{00}(x)\right\rangle
$$

and the specific heat is then given by

$$
\mathcal{C}_{v}=\left(\frac{d \mathcal{U}}{d T}\right)_{\mathcal{N}}
$$

Here the temperature derivative has to be taken at constant particle number density, in contrast with the calculation of the low temperature specific heat in the previous section, where all temperature derivatives were taken at constant chemical potential, see Eq. (6). In [14] this fact was mentioned as explanation for the disagreement with the previous calculation, but, as Eq. (44) makes clear, this could only affect terms of order $T^{3}$ in the low-temperature expansion. Indeed, we shall show now that a complete calculation based on the internal energy also leads to a $g^{2} T \ln T^{-1}$ in the specific heat.

In 14] the specific heat is computed using the following formula for the total energy density,

$$
\mathcal{U}=2 \int d \omega \int \frac{d^{3} k}{(2 \pi)^{3}} n_{f}(\omega) \omega \rho_{+}(\omega, k)
$$

where $\rho_{+}$is the spectral density of the positive energy component of the quark propagator (see below). It should be noted that this formula is incorrect even for a theory with only instantaneous interactions of the type

$$
H_{i n t}=\frac{1}{2} \int d^{3} x d^{3} x^{\prime} \psi_{\alpha}^{\dagger}(\mathbf{x} t) \psi_{\beta}^{\dagger}\left(\mathbf{x}^{\prime} t\right) V_{\alpha \alpha^{\prime}, \beta \beta^{\prime}}\left(\mathbf{x}-\mathbf{x}^{\prime}\right) \psi_{\beta^{\prime}}\left(\mathbf{x}^{\prime} t\right) \psi_{\alpha^{\prime}}(\mathbf{x} t),
$$

in which case the correct formula reads [29]

$$
\mathcal{U}=2 \int d \omega \int \frac{d^{3} k}{(2 \pi)^{3}} n_{f}(\omega) \frac{1}{2}(\omega+k) \rho_{+}(\omega, k) .
$$

The anomalous behaviour of the specific heat comes from dynamically screened interactions, whose non-instantaneous character cannot be neglected. It might be rather difficult to generalize Eq. (32) directly for non-instantaneous interactions, because one would have to use an effective Hamiltonian which is nonlocal in time. Therefore, we will use the full energy momentum tensor of QCD without integrating out the gluons.

The energy momentum tensor can be written as a sum of three distinct pieces,

$$
T^{\mu \nu}=T_{(\mathrm{q})}^{\mu \nu}+T_{(\mathrm{g})}^{\mu \nu}+T_{(\text {int. })}^{\mu \nu},
$$

corresponding to the quark part, the gluon part, and the interaction part. The contributions of these parts will be evaluated in the following subsections. We will neglect gluon self interactions and ghost contributions, since they give only higher order corrections at low temperatures. 


\section{A. Quark part}

The quark part is given by

$$
T_{(\mathrm{q})}^{00}=i \sum_{f} \bar{\psi} \gamma^{0} \partial^{0} \psi
$$

where we have written explicitly the sum over flavor space. This is the (only) contribution which is taken into account by Boyanovsky and de Vega [14]. We now repeat their calculation, but for simplicity without the renormalization group improvement of the quark propagator proposed in 14]. Taking into account only the positive energy component of the quark propagator, we find

$$
\mathcal{U}_{(\mathrm{q})}=2 N N_{f} \int d \omega \int \frac{d^{3} k}{(2 \pi)^{3}} n_{f}(\omega) \omega \rho_{+}(\omega, k),
$$

where the spectral density is defined as $\rho_{+} \equiv \frac{1}{\pi} \operatorname{Im} S_{+}$.

In order to obtain the specific heat from Eq. (29), we have to determine first the temperature dependence of the chemical potential from the condition

$$
\frac{d \mathcal{N}}{d T} \equiv \frac{\partial \mathcal{N}}{\partial T}+\frac{d \mu}{d T} \frac{\partial \mathcal{N}}{\partial \mu}=0
$$

where the particle number density $\mathcal{N}$ is given by

$$
\mathcal{N}=2 N N_{f} \int d \omega \int \frac{d^{3} k}{(2 \pi)^{3}} n_{f}(\omega) \rho_{+}(\omega, k)
$$

(up to anti-particle contributions.) We expand $\mathcal{N}$ with respect to $g$,

$$
\mathcal{N}=\mathcal{N}_{0}+g_{\text {eff }}^{2} \mathcal{N}_{2}+\ldots
$$

The free contribution $\mathcal{N}_{0}$ is given by

$$
\mathcal{N}_{0}=N N_{f}\left(\frac{\mu^{3}}{3 \pi^{2}}+\frac{\mu T^{2}}{3}\right) \text {. }
$$

In $\mathcal{N}_{2}$ we are only interested in contributions which contain $\ln (M / T)$. Such terms arise from infrared singularities caused by the transverse gluon propagator, which are dynamically screened. This corresponds to scattering processes of quarks which are close to the Fermi surface. Therefore the anomalous terms come from the region $k \sim \omega \sim \mu$, where $\Sigma_{+}$is given by (9). Subtracting the temperature independent part, we find then

$$
g_{\text {eff }}^{2} \mathcal{N}_{2}=\left.2 N N_{f} \int d \omega \int \frac{d^{3} k}{(2 \pi)^{3}}\left(n_{f}(\omega)-\theta(\mu-\omega)\right) \delta\left(\omega-k+\operatorname{Re} \Sigma_{+}\right)\right|_{\mathcal{O}\left(g_{\text {eff }}^{2}\right)},
$$

where we have approximated the spectral density by a delta function, since the imaginary part of $\Sigma_{+}$turns out to be negligible compared to its real part. The integration can be performed easily, with the result

$$
g_{\mathrm{eff}}^{2} \mathcal{N}_{2} \simeq \frac{g_{\mathrm{eff}}^{2} N_{g} \mu T^{2}}{36 \pi^{2}} \ln \left(\frac{M}{T}\right)
$$

We notice that this result is consistent with the result for the entropy, Eq. (25). Now we can solve Eq. (36) at low temperature,

$$
\frac{d \mu}{d T}=-\frac{2 \pi^{2} T}{3 \mu}-\frac{g^{2} C_{f} T}{18 \mu} \ln \left(\frac{M}{T}\right)
$$


The approximate solution to this differential equation is given by

$$
\mu(T) \simeq \mu(0)\left(1-\frac{\pi^{2} T^{2}}{3 \mu(0)^{2}}-\frac{g^{2} C_{f} T^{2}}{36 \mu(0)^{2}} \ln \left(\frac{M}{T}\right)\right) .
$$

Eqs. (42) and (43) correctly reproduce the beginning of the perturbative expansions of the corresponding formulae in [14] (Eqs. (2.37), (2.38)).

For the specific heat we obtain from Eq. (35), following the same steps as in the calculation of $d \mathcal{N} / d T$

$$
C_{v(\mathrm{q})} \simeq N N_{f} \mu^{2} T+N N_{f} \frac{\mu^{3}}{\pi^{2}} \frac{d \mu}{d T}+\frac{g_{\mathrm{eff}}^{2} N_{g} \mu^{2} T}{18 \pi^{2}} \ln \left(\frac{M}{T}\right)
$$

Using Eqs. (42) and (43) we find that the $T \ln T$-terms cancel,

$$
C_{v(\mathrm{q})} \simeq N N_{f} \frac{\mu^{2} T}{3}+O\left(T^{3}\right),
$$

as stated in 14]. We should emphasize that this cancellation has nothing to do with the nonperturbative renormalization group method which is employed in 14] (and which has recently been criticized in Ref. [30]).

The authors of Ref. 14] also determined a contribution which prior to the renormalization group improvement corresponds to a term of order $g^{2} T^{3} \ln (M / T)$. This type of nonanalytic terms however appears already in regular Fermi-liquids 31] and moreover is subleading to ordinary perturbative corrections $g^{2} \mu^{2} T$ at low $T$, which have not been evaluated in [14].

\section{B. Gluon part}

We now turn to the gluon part of the energy density, which has explicitly been neglected in Ref. [14]. This is given by

$$
T_{(\mathrm{g})}^{00}=\frac{1}{2}\left(\mathbf{E}^{a} \cdot \mathbf{E}^{a}+\mathbf{B}^{a} \cdot \mathbf{B}^{a}\right) .
$$

Neglecting gluon self interactions, and keeping only the transverse part of the gluon propagator, we obtain

$$
\mathcal{U}_{\text {(g) }} \simeq 2 N_{g} \int \frac{d^{4} K}{(2 \pi)^{4}} n_{b}(\omega) \operatorname{Im}\left(\left(\omega^{2}+k^{2}\right) D_{T}\right)
$$

The pole contribution to this integral is again exponentially suppressed, therefore we only have to consider the cut contribution. At low temperature the temperature dependence of the gluon self energy can be neglected and we find

$$
\mathcal{U}_{(\mathrm{g})} \simeq 2 N_{g} \int \frac{d^{3} K}{(2 \pi)^{3}} \int_{0}^{\infty} \frac{d \omega}{\pi} n_{b}(\omega)\left(\omega^{2}+k^{2}\right) \frac{\frac{g_{\mathrm{eff}}^{2} \mu^{2} \omega}{4 \pi k}}{\left(\omega^{2}-k^{2}\right)^{2}+\left(\frac{g_{\mathrm{eff}}^{2} \mu^{2} \omega}{4 \pi k}\right)^{2}},
$$

where we have dropped less infrared-sensitive contributions not involving the Bose distribution. After the substitution $\omega=T y, k^{3}=g_{\text {eff }}^{2} \mu^{2} T x /(4 \pi)$ the integral can be readily done, with the result

$$
\mathcal{U}_{(\mathrm{g})} \simeq \frac{g_{\mathrm{eff}}^{2} N_{g} \mu^{2} T^{2}}{72 \pi^{2}} \ln (M / T)
$$


which gives the following contribution to the specific heat at order $T \ln T$,

$$
C_{v(\mathrm{~g})} \simeq \frac{g_{\mathrm{eff}}^{2} N_{g} \mu^{2} T}{36 \pi^{2}} \ln (M / T) .
$$

Again the determination of the constant $M$ would require a more accurate calculation, similar to the one in section IV]

\section{Interaction part}

The interaction part is given by

$$
T_{\text {(int) }}^{00}=g \sum_{f} \bar{\psi} \gamma^{0} A_{a}^{0} T_{a} \psi
$$

The expectation value of this term is essentially given by the resummed gluon ring diagram as in 15]. However, here only the longitudinal component of the gluon propagator appears in the loop. This mode is subject to Debye screening, so it can contribute only to the normal Fermi liquid part of the specific heat.

\section{Result}

We have thus found that the only contribution to the specific heat at order $T \ln T$ when calculated along the lines of Ref. 14] comes from the gluon part, Eq. (50). While this confirms the observation of Ref. [14] that the quark contribution of order $g^{2} T \ln T$ cancels against a similar term in the temperature dependence of the chemical potential at fixed number density, it shows that the (explicit) neglect of the gluon contribution to the internal energy in [14] is not justified.

Curiously enough, the gluon contributions can be neglected when calculating the anomalous specific heat from the entropy functional (6). But the calculation of section 1 can also be viewed as receiving a net contribution only from a purely gluonic term (26), since the anomalous contribution contained in the $S_{(\Pi)}$ part is equal but opposite in sign to the fermionic part $S_{(q)}$. In this respect the calculation based on the internal energy is perfectly in line with the calculation based on the entropy. The different possibilities for organizing the calculation of the anomalous contribution to the specific heat thus correspond to 'integrating out' first the fermionic degrees of freedom or first the gluonic ones.

\section{HIGHER ORDERS IN THE LOW-TEMPERATURE SPECIFIC HEAT}

In this section we shall evaluate higher terms in the low-temperature expansion of the specific heat which go beyond the leading log approximation. A convenient starting point is the following expression for the pressure, which can be viewed as the result of having integrated out first the fermionic degrees of freedom, and which thus concentrates on the effects of the only dynamically screened transverse gauge bosons in the low-temperature expansion of the thermodynamic potential:

$$
\begin{aligned}
P & =N N_{f}\left(\frac{\mu^{4}}{12 \pi^{2}}+\frac{\mu^{2} T^{2}}{6}+\frac{7 \pi^{2} T^{4}}{180}\right) \\
& -N_{g} \int \frac{d^{3} q}{(2 \pi)^{3}} \int_{0}^{\infty} \frac{d q_{0}}{\pi}\left[2\left(\left[n_{b}+\frac{1}{2}\right] \operatorname{Im} \ln D_{T}^{-1}-\frac{1}{2} \operatorname{Im} \ln D_{\text {vac }}^{-1}\right)\right.
\end{aligned}
$$




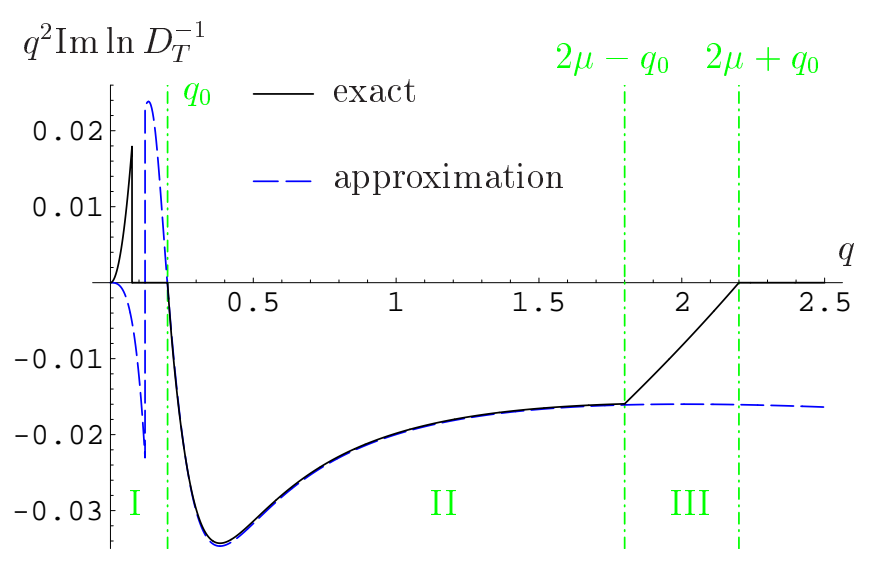

FIG. 1: Integrand for the $q$-integration $q^{2} \operatorname{Im} \ln \left(q^{2}-q_{0}^{2}+\Pi_{T}+\Pi_{\mathrm{vac}}\right)$ for $\mu=\bar{\mu}_{\mathrm{MS}} / 2=1, q_{0}=0.2, g_{\mathrm{eff}}^{2}=1$. The solid line shows the exact result that follows from the full one-loop self energy expressions at $T=0$, the dashed line shows the result with the approximations of Eqs. (56) and (57). The parameter $q_{0}=0.2$ is chosen this large as to clearly show the three different ranges. As discussed in the text, the main contribution only comes from region II.

$$
\left.+\left(\left[n_{b}+\frac{1}{2}\right] \operatorname{Im} \ln \frac{D_{L}^{-1}}{q^{2}-q_{0}^{2}}-\frac{1}{2} \operatorname{Im} \ln \frac{D_{\mathrm{vac}}^{-1}}{q^{2}-q_{0}^{2}}\right)\right]++O\left(g^{2} T^{4}\right)+O\left(g^{4} \mu^{4}\right) .
$$

Here the inverse gauge boson propagators are given by $D_{T}^{-1}=q^{2}-q_{0}^{2}+\Pi_{T}+\Pi_{\mathrm{vac}}, D_{L}^{-1}=$ $q^{2}-q_{0}^{2}+\Pi_{L}+\Pi_{\mathrm{vac}}$, and $D_{\mathrm{vac}}^{-1}=q^{2}-q_{0}^{2}+\Pi_{\mathrm{vac}}$, where $\Pi_{T, L, v a c}$ are the matter and vacuum contributions to the gauge boson self-energy produced by an undressed one-loop fermion diagram. This expression becomes exact in the limit of large flavor number $N_{f}$ and has been studied in Refs. 15, 16] and used to test the behavior of perturbation theory at finite temperature and chemical potential. At finite $N_{f}$, Eq. (52) with $\Pi$ including also the leading contributions from gluon loops still collects all infrared-sensitive contributions up to and including three-loop order 32]. We shall however find that all contributions from gluon loops to $\Pi$ enter the specific heat only at and beyond order $g_{\mathrm{eff}}^{2} T^{3}$, and thus will be negligible for $T \ll \mu$ compared to the terms we shall keep.

In the following we will always drop temperature independent terms in the pressure, since they do not contribute to the specific heat at low temperature.

\section{A. Transverse contribution}

The $n_{b}$-part of the contribution of the transverse gluons to the pressure is given by

$$
\frac{P_{T}}{N_{g}}=-2 \int \frac{d^{3} q}{\left(2 \pi^{3}\right)} \int_{0}^{\infty} \frac{d q_{0}}{\pi} n_{b} \operatorname{Im} \ln \left(q^{2}-q_{0}^{2}+\Pi_{T}+\Pi_{\mathrm{vac}}\right) .
$$

As long as $T \ll \mu$, it is sufficient to take the self energy at zero temperature, which is given by a single fermion loop. From the explicit form of the self energy [33] we see that the $q$-integration naturally splits into three regions: $q<q_{0}$ (I), $q_{0}<q<2 \mu-q_{0}$ (II), and $2 \mu-q_{0}<q<2 \mu+q_{0}$ (III), see Fig. 1.

Usually region I contains the ideal-gas pressure of the gluons, $\pi^{2} T^{4} / 45$ per gluon, and perturbative corrections $\propto g_{\text {eff }}^{2} \mu^{2} T^{2}$. However, at low temperatures $T \ll \omega_{p} \sim g_{\text {eff }} \mu$, these contributions are suppressed by a factor $e^{-\omega_{p} / T}$ which goes to zero with all derivatives vanishing and thus do not 
contribute to the low-temperature series. All other contributions from region I are suppressed by further powers of $g_{\text {eff }}$.

In region III we may expand the self energy about $q=2 \mu$ and $q_{0}=0$. Then one finds that this contribution is of higher order in $g_{\text {eff }}$. We conclude that we may restrict our attention to the region II.

In region II the Bose-Einstein factor and the leading term in the gluon self energy set the characteristic scales. Since $q_{0} \sim T \ll g_{\text {eff }} \mu$, this is dominated by the well-known 34] dynamical screening pole at imaginary $q$ with $|q| \simeq\left(g_{\text {eff }}^{2} \mu^{2} q_{0} /(4 \pi)\right)^{1 / 3}$ and we have

$$
q_{0} \sim T, \quad q \sim\left(g_{\text {eff }}^{2} \mu^{2} T\right)^{1 / 3}
$$

in the infrared-sensitive part of region II.

We shall therefore perform an expansion with respect to a parameter $b$ defined by

$$
b:=\left(\frac{T}{g_{\mathrm{eff}} \mu}\right)^{1 / 3} .
$$

It turns out that the following approximation of the gluon self energy is sufficient through order $T^{3} \ln T$ in the entropy (see Fig. 1),

$$
\begin{aligned}
& \operatorname{Re} \Pi_{T}\left(q_{0}, q\right) \simeq \frac{g_{\text {eff }}^{2}}{\pi^{2}}\left(\frac{\mu^{2} q_{0}^{2}}{q^{2}}-\frac{\mu^{2} q_{0}^{4}}{3 q^{4}}\right), \\
& \operatorname{Im} \Pi_{T}\left(q_{0}, q\right) \simeq \frac{g_{\text {eff }}^{2}}{4 \pi}\left(-\frac{\mu^{2} q_{0}}{q}+\frac{\mu^{2} q_{0}^{3}}{q^{3}}-\frac{q q_{0}}{4}\right) .
\end{aligned}
$$

The first two terms in both lines are the leading terms of an expansion of the HDL self energy 24, 25, 26, 27], in powers of $q_{0}$. Naively counting powers of $b$ in the integrand one would conclude that only these terms are responsible for the terms of order $b^{6}$ through $b^{12}$ in the pressure (perhaps with additional factors of $\ln b$ ). In principle this is correct, but one should keep in mind that the integration limits of the $q$-integration depend on $\mu$ and $q_{0}$, which might invalidate a naive power counting. However, it turns out that there is only one instance where a term which is formally suppressed in the naive power counting scheme has to be included in the self energy (see [36] for a rigorous proof): It is the term of order $b^{6}$ in the pressure, where one also finds a contribution from the last term in Eq. (57), which is beyond the HDL approximation (but it is still included in the large- $N_{f}$ limit.) The $b^{6}$ term plays a special role anyway, as this is the only term where we also get a contribution from the non- $n_{b}$-part (see below).

Introducing dimensionless integration variables $x$ and $y$ via $q_{0}=b^{3} g_{\text {eff }} \mu y$ and $q=$ $b g_{\text {eff }} \mu(x /(4 \pi))^{1 / 3}$, we find after expanding the integrand with respect to $b$,

$$
\begin{aligned}
& \frac{P_{T}^{\mathrm{II}}}{N_{g}} \simeq \frac{g_{\mathrm{eff}}^{4} \mu^{4}}{12 \pi^{4}} \int_{0}^{\infty} d y \frac{1}{e^{y}-1} \int_{x_{\text {min }}}^{x_{\text {max }}} d x\left[b^{6} \arctan \left(\frac{y}{x}\right)\right. \\
& +\frac{y\left(g_{\mathrm{eff}}^{2} x^{2}-64 y^{2}\right)}{8\left(2 \pi^{2} x\right)^{1 / 3}\left(x^{2}+y^{2}\right)} b^{8}+\frac{32(2 x)^{1 / 3} y^{5}}{\pi^{4 / 3}\left(x^{2}+y^{2}\right)^{2}} b^{10} \\
& \left.-\frac{32 y^{5}\left(24 x^{2} y^{2}-8 y^{4}+\pi^{2}\left(x^{2}+y^{2}\right)^{2}\right)}{3 \pi^{2} x\left(x^{2}+y^{2}\right)^{3}} b^{12}+O\left(b^{14}\right)\right],
\end{aligned}
$$

with $x_{\min }=4 \pi b^{6} y^{3}$ and $x_{\max }=4 \pi\left(2-b^{3} g_{\mathrm{eff}} y\right)^{3} /\left(b g_{\mathrm{eff}}\right)^{3}$. In the coefficients of this expansion we have written down only those terms which do not ultimately lead to terms that are suppressed by 
explicit positive powers of $g_{\text {eff }}$. The integrations are now straightforward, and we find

$$
\begin{gathered}
\frac{P_{T}^{\mathrm{II}} \simeq}{N_{g}} g_{\mathrm{eff}}^{4} \mu^{4}\left[\frac{b^{6}}{72 \pi^{2}}\left(\ln \left(\frac{32 \pi}{\left(b g_{\mathrm{eff}}\right)^{3}}\right)+\gamma_{E}-\frac{6}{\pi^{2}} \zeta^{\prime}(2)+\frac{3}{2}\right)-\frac{2^{2 / 3} \Gamma\left(\frac{8}{3}\right) \zeta\left(\frac{8}{3}\right)}{3 \sqrt{3} \pi^{11 / 3}} b^{8}\right. \\
\left.+\frac{82^{1 / 3} \Gamma\left(\frac{10}{3}\right) \zeta\left(\frac{10}{3}\right)}{9 \sqrt{3} \pi^{13 / 3}} b^{10}+\frac{16\left(\pi^{2}-8\right)}{45 \pi^{2}} b^{12} \ln b+\tilde{c}_{T} b^{12}+O\left(b^{14}\right)\right] .
\end{gathered}
$$

The evaluation of the constant $\tilde{c}_{T}$ is a bit more involved because one has to sum up an infinite series of contributions from the infrared region. This calculation is performed in the Appendix and leads to an integral representation of $\tilde{c}_{T}$ that we have been able to evaluate only numerically, with the result $\tilde{c}_{T}=-0.001786743 \ldots$

\section{B. Longitudinal contribution}

The $n_{b}$-part of the contribution of the longitudinal gluons to the pressure is given by

$$
\frac{P_{L}}{N_{g}}=-\int \frac{d^{3} q}{\left(2 \pi^{3}\right)} \int_{0}^{\infty} \frac{d q_{0}}{\pi} n_{b} \operatorname{Im} \ln \left(\frac{q^{2}-q_{0}^{2}+\Pi_{L}+\Pi_{\mathrm{vac}}}{q^{2}-q_{0}^{2}}\right) .
$$

As in the previous section the $q$-integration decomposes into three parts.

Again, contributions from region III are suppressed by explicit powers of $g_{\text {eff }}$ compared to those of the other regions.

The dominant contribution comes from region II, $q_{0}<q<2 \mu-q_{0}$. Now the characteristic scales are

$$
q_{0} \sim T, \quad q \sim g_{\text {eff }} \mu,
$$

because of Debye screening of the longitudinal gluons with leading-order mass $m_{D}=g_{\text {eff }} \mu / \pi$. In a similar way as in the previous section, the gluon self energy can be approximated as

$$
\begin{aligned}
& \operatorname{Re} \Pi_{L}\left(q_{0}, q\right) \simeq \frac{g_{\text {eff }}^{2}}{\pi^{2}}\left(\mu^{2}-\frac{2 \mu^{2} q_{0}^{2}}{q^{2}}-\frac{q^{2}}{12}\right), \\
& \operatorname{Im} \Pi_{L}\left(q_{0}, q\right) \simeq \frac{g_{\text {eff }}^{2}}{2 \pi}\left(\frac{\mu^{2} q_{0}}{q}-\frac{\mu^{2} q_{0}^{3}}{q^{3}}-\frac{q q_{0}}{4}\right) .
\end{aligned}
$$

We introduce dimensionless integration variables $y$ and $z$ via $q_{0}=b^{3} g_{\text {eff }} \mu y, q=g_{\text {eff }} \mu z / \pi$. Then we find after expanding the integrand with respect to $b$,

$$
\begin{aligned}
& \frac{P_{L}^{\mathrm{II}}}{N_{g}} \simeq \frac{g_{\mathrm{eff}}^{4} \mu^{4}}{16 \pi^{2}} \int_{0}^{\infty} d y \frac{1}{e^{y}-1} \int_{z_{\min }}^{z_{\max }} d z \\
& \times\left[b^{6} \frac{y z\left(-4 \pi^{2}\left(1+z^{2}\right)+g_{\mathrm{eff}}^{2} z^{4}\right)}{\pi^{4}\left(1+z^{2}\right)^{2}}+b^{12} \frac{y^{3}\left(\pi^{2}-12\left(z^{2}+1\right)\right)}{3 z\left(1+z^{2}\right)^{3}}+O\left(b^{18}\right)\right],
\end{aligned}
$$

with $z_{\min }=b^{3} y \pi$ and $z_{\max }=\left(2 / g_{\text {eff }}-b^{3} y\right) \pi$. In the coefficients of this expansion we have written down only those terms which do not lead ultimately to terms that are suppressed by explicit positive powers of $g_{\text {eff }}$. The integrations are now straightforward, and we find

$$
\frac{P_{L}^{\mathrm{II}}}{N_{g}} \simeq g_{\mathrm{eff}}^{4} \mu^{4}\left[\frac{b^{6}}{48 \pi^{2}}\left(1+\ln \left(\frac{g_{\mathrm{eff}}^{2}}{4 \pi^{2}}\right)\right)+\frac{\pi^{2}\left(12-\pi^{2}\right)}{240} b^{12} \ln b+\tilde{c}_{L}^{\mathrm{II}} b^{12}+O\left(b^{18} \ln b\right)\right] .
$$


The constant $\tilde{c}_{L}^{\mathrm{II}}$ can be determined by summing up IR enhanced contributions in analogy to the constant $\tilde{c}_{T}$ of Sect. IVA and its integral representation is given in Eq. (A8). Numerically, we get $\tilde{c}_{L}^{\mathrm{II}} \approx 0.11902569216 \ldots$

In contrast to the case of transverse polarizations, there is now however also a contribution from region I, of the order of $g_{\text {eff }}^{4} \mu^{4} b^{12}=T^{4}$. The term involving $\operatorname{Im} \ln \left(q^{2}-q_{0}^{2}+\Pi_{L}+\Pi_{\mathrm{vac}}\right)$ is again exponentially suppressed for $T \ll \omega_{p} \sim g_{\text {eff }} \mu$. However, at these temperatures the term involving $\operatorname{Im} \ln \left(q^{2}-q_{0}^{2}\right)$ contributes the equivalent of an ideal-gas pressure of one bosonic degree of freedom, but with negative sign, leading to

$$
\frac{P_{L}^{\mathrm{I}}}{N_{g}} \simeq-\frac{\pi^{2} T^{4}}{90}+O\left(e^{-\omega_{p} / T}\right)
$$

so that $\tilde{c}_{L}=\tilde{c}_{L}^{\mathrm{II}}-\pi^{2} T^{4} / 90=0.009363421 \ldots$

\section{Non- $n_{b}$ contribution}

The nonanalytic terms in the low-temperature expansion of Eq. (52) all come from the parts of the integrals involving the Bose distribution $n_{b}$. The non- $n_{b}$ parts in Eq. (152) are less IR singular and can be calculated by expanding out the self energy diagrams. We can determine their contribution by the observation that at two-loop order also the $n_{b}$ part is IR safe and given by

$$
\frac{P_{n_{b}}^{2-\text { loop }}}{N_{g}}=-\int \frac{d^{3} q}{(2 \pi)^{3}} \int_{0}^{\infty} \frac{d q_{0}}{\pi} n_{b} \operatorname{Im}\left(\frac{2 \Pi_{T}+\Pi_{L}}{q^{2}-q_{0}^{2}-i \epsilon}\right) .
$$

In this integral we have two contributions, one from the real parts and one from the imaginary parts of the gluon self energies. One finds that these two contributions cancel precisely at the order $g_{\text {eff }}^{2} \mu^{2} T^{2}$. (As above one finds that the $T=0$ gluon self energies are sufficient at this order.) Therefore the two-loop non- $n_{b}$ contribution has to be equal to the standard perturbative result at order $g_{\text {eff }}^{2} \mu^{2} T^{2}$ [35],

$$
\frac{1}{N_{g}}\left[P_{\mathrm{non}-n_{b}}-\left.P_{\mathrm{non}-n_{b}}\right|_{T=0}\right]=-\frac{g_{\mathrm{eff}}^{2} \mu^{2} T^{2}}{16 \pi^{2}}+\mathcal{O}\left(g_{\mathrm{eff}}^{2} T^{4}\right) .
$$

\section{Combined result}

Our final expression for the leading temperature-dependent contribution to the interaction pressure in the regime $T \ll g \mu$ is contained in

$$
\Delta P=P-P^{0}=P_{T}^{\mathrm{II}}+P_{L}^{\mathrm{I}}+P_{L}^{\mathrm{II}}+P_{\mathrm{non}-n_{b}}-P^{0}+O\left(g^{4} \mu^{4}\right),
$$

where $P^{0}$ is the ideal-gas pressure, and explicitly reads

$$
\begin{aligned}
\frac{1}{N_{g}}\left[\Delta P-\left.\Delta P\right|_{T=0}\right]= & \frac{g_{\mathrm{eff}}^{2} \mu^{2} T^{2}}{72 \pi^{2}}\left(\ln \left(\frac{4 g_{\mathrm{eff}} \mu}{\pi^{2} T}\right)+\gamma_{E}-\frac{6}{\pi^{2}} \zeta^{\prime}(2)-\frac{3}{2}\right) \\
& -\frac{2^{2 / 3} \Gamma\left(\frac{8}{3}\right) \zeta\left(\frac{8}{3}\right)}{3 \sqrt{3} \pi^{11 / 3}} T^{8 / 3}\left(g_{\mathrm{eff}} \mu\right)^{4 / 3}+8 \frac{2^{1 / 3} \Gamma\left(\frac{10}{3}\right) \zeta\left(\frac{10}{3}\right)}{9 \sqrt{3} \pi^{13 / 3}} T^{10 / 3}\left(g_{\mathrm{eff}} \mu\right)^{2 / 3} \\
& +\frac{2048-256 \pi^{2}-36 \pi^{4}+3 \pi^{6}}{2160 \pi^{2}} T^{4}\left[\ln \left(\frac{g_{\mathrm{eff}} \mu}{T}\right)+\bar{c}\right] \\
& +O\left(T^{14 / 3} /\left(g_{\mathrm{eff}} \mu\right)^{2 / 3}\right)+O\left(g^{4} \mu^{2} T^{2} \ln T\right)
\end{aligned}
$$


where the constant $\bar{c}$ is given by

$$
\begin{aligned}
\bar{c}=\gamma_{E} & -90 \frac{\zeta^{\prime}(4)}{\pi^{4}}-\frac{31}{12}+\frac{1}{2048-256 \pi^{2}-36 \pi^{4}+3 \pi^{6}} \times \\
\times & \left\{3 \pi^{4}\left(12-\pi^{2}\right) \ln \pi+128\left(\pi^{2}-8\right) \ln (4 \pi)+3 \pi^{2}\left(29 \pi^{2}+32\right)\right. \\
& -72 \pi^{3} \int_{1}^{\infty} d z\left[\frac{1024+\pi^{6}-64 \pi^{2}\left(2+3 z^{2}\right)+12 \pi^{4}\left(-1-z^{2}+3 z^{3}\right)}{24 \pi^{3} z}\right. \\
& \left.\left.+2 z^{2} \arctan \left[\frac{\pi\left(1-z^{2}\right)}{2 z+\left(z^{2}-1\right) \ln \left(\frac{z+1}{z-1}\right)}\right]+z^{2} \arctan \left[-\frac{2 z}{\pi}+\frac{1}{\pi} \ln \left(\frac{z+1}{z-1}\right)\right]\right]\right\} \\
\approx & 4.099348512039 \ldots
\end{aligned}
$$

The terms involving logarithms and fractional powers of $T$ all come from the cut contribution of region II, whereas $P^{\mathrm{I}}-P^{0}=-\frac{3}{2} P^{0}+O\left(e^{-\omega / T}\right)$ only contributes to $\bar{c}$.

From Eq. (70) one can obtain the entropy density through $\mathcal{S}=(\partial P / \partial T)_{\mu}$ and the specific heat through [18]

$$
\mathcal{C}_{v}=T\left(\left(\frac{\partial \mathcal{S}}{\partial T}\right)_{\mu}-\left(\frac{\partial \mathcal{N}}{\partial T}\right)_{\mu}^{2}\left(\frac{\partial \mathcal{N}}{\partial \mu}\right)_{T}^{-1}\right),
$$

with $\mathcal{N}=(\partial P / \partial \mu)_{T}$, which in the ideal-gas limit reads

$$
\mathcal{C}_{v}^{0}=N N_{f}\left[\frac{\mu^{2} T}{3}+T^{3}\left(\frac{7 \pi^{2}}{15}-\frac{4 \mu^{2}}{3 T^{2}+9 \mu^{2} / \pi^{2}}\right)\right]+N_{g} \frac{4 \pi^{2} T^{3}}{15} .
$$

For the interaction part of the specific heat only the logarithmic derivative of the entropy in formula (72) contributes, and is given explicitly by

$$
\begin{aligned}
& \frac{\mathcal{C}_{v}-\mathcal{C}_{v}^{0}}{N_{g}}=\frac{g_{\mathrm{eff}}^{2} \mu^{2} T}{36 \pi^{2}}\left(\ln \left(\frac{4 g_{\mathrm{eff}} \mu}{\pi^{2} T}\right)+\gamma_{E}-\frac{6}{\pi^{2}} \zeta^{\prime}(2)-3\right) \\
& \quad-40 \frac{2^{2 / 3} \Gamma\left(\frac{8}{3}\right) \zeta\left(\frac{8}{3}\right)}{27 \sqrt{3} \pi^{11 / 3}} T^{5 / 3}\left(g_{\mathrm{eff}} \mu\right)^{4 / 3}+560 \frac{2^{1 / 3} \Gamma\left(\frac{10}{3}\right) \zeta\left(\frac{10}{3}\right)}{81 \sqrt{3} \pi^{13 / 3}} T^{7 / 3}\left(g_{\mathrm{eff}} \mu\right)^{2 / 3} \\
& \quad+\frac{2048-256 \pi^{2}-36 \pi^{4}+3 \pi^{6}}{180 \pi^{2}} T^{3}\left[\ln \left(\frac{g_{\mathrm{eff}} \mu}{T}\right)+\bar{c}-\frac{7}{12}\right] \\
& \quad+O\left(T^{11 / 3} /\left(g_{\mathrm{eff}} \mu\right)^{2 / 3}\right)+O\left(g^{4} \mu^{2} T \ln T\right) .
\end{aligned}
$$

We remark that in Eq. (74) one could replace $\mu$ with $\mu(T)$, as given in Eq. (43), as this would modify the result only beyond the terms of order $T^{3}$ since $T /(g \mu) \ll 1$.

Using the method described in Sect. IVA-IVC, one can in principle compute the coefficients of higher terms in the expansion of $\mathcal{C}_{v}$ with respect to $b$. This is straightforward for the coefficients of the fractional powers and the logarithmic terms, where one only has to include higher orders in the expansion of the HDL gluon self energy with respect to $q_{0}$, see Eqs. (56), (57), (62), (63). For the terms of order $T^{5}, T^{7}, T^{9}$ etc., however, one has to sum up again IR enhanced terms, in a similar way as in the calculation of $\tilde{c}$ described in the Appendix.

The low-temperature expansion that we have carried out has assumed that $T \ll g_{\text {eff }} \mu$ as well as $g_{\text {eff }} \ll 1$. If we set $T / \mu \sim g_{\text {eff }}^{1+\delta}$ with $\delta>0$, we find that the terms in the expansion (74) correspond to the orders $g_{\mathrm{eff}}^{3+\delta} \ln \left(c / g_{\mathrm{eff}}\right), g_{\mathrm{eff}}^{3+(5 / 3) \delta}, g_{\mathrm{eff}}^{3+(7 / 3) \delta}$, and $g_{\mathrm{eff}}^{3+3 \delta} \ln \left(c / g_{\mathrm{eff}}\right)$, respectively, with 
a truncation error of the order $g_{\mathrm{eff}}^{3+(11 / 3) \delta}$ from higher terms in the ring diagrams. We have neglected perturbative corrections to these terms, which at a minimum arise at the order $g_{\text {eff }}^{5+\delta} \ln \left(c / g_{\text {eff }}\right)$.

One might suspect that higher order terms could involve also higher powers of $g_{\text {eff }}^{2} \ln T$, which could resum into a leading term $\mu^{2} T^{1+O\left(g_{\text {eff }}^{2}\right)}$. However, it has been argued in [3] that the leading $g_{\text {eff }}^{2} T \ln T^{-1}$ is not modified by higher order corrections in QED, and this has been corroborated recently by the authors of Ref. [30] using a high-density effective field theory also applicable to QCD. It can therefore be expected that the leading term in the above low-temperature series remains valid even when the temperature is so low that $g_{\text {eff }}^{2} \ln \left(g_{\text {eff }} \mu / T\right) \gg 1$.

On the other hand, the higher terms of the low-temperature expansion involving fractional powers $T^{(2 n+3) / 3}$ with $n \geq 1$ remain more important than the undetermined perturbative corrections (which are suppressed by explicit powers of $g_{\text {eff }}^{2}$ ) only when $\delta<3 / n$.

\section{HDL RESUMMATION}

As we have seen in Sect. IV the nonanalytic terms in the low-temperature expansion of the thermodynamic potential are determined by HDL contributions to the gluon self-energy. Terms beyond the HDL approximation are relevant for contributions from hard momenta $q \sim \mu$, yielding a term of order $g_{\text {eff }}^{2} \mu^{2} T^{2}$ in the temperature-dependent part of the pressure. However, this is a perturbative piece that can be identified as a two-loop contribution without the need for resummations. When this contribution is subtracted from the full one-loop expression, the remainder is dominated by soft momenta $q \ll \mu$ and the HDL approximation is sufficient.

In this section we shall consider the full HDL-resummed ring diagrams, which allows us to relax the requirement $T \ll g_{\text {eff }} \mu$, under which the above low-temperature series is meaningful, to only $T \ll \mu$. When expanded around $T=0$, the one-loop HDL-resummed thermodynamic potential contains all the anomalous terms of the low-temperature series (70). However, as we have already seen there are also terms from region I which behave as $\sim e^{-\omega_{p} / T}$ and thus do not show up at any finite order of the low-temperature series. Nevertheless, such terms become important for $T \sim g_{\text {eff }} \mu$. By numerically evaluating the full HDL-resummed expression we can capture their effect as well and study the behavior of entropy and specific heat for all temperatures $T \ll \mu$.

\section{A. Separation of hard and soft contributions}

In the transverse sector, the one contribution in Eq. (58) from a non-HDL term in the gluon self-energy can also be written as

$$
\begin{aligned}
& \frac{1}{N_{g}}\left[P_{T}^{\mathrm{II}, \text { non }-\mathrm{HDL}}-\left.P_{T}^{\mathrm{II}, \text { non-HDL }}\right|_{T=0}\right] \\
& =-\frac{1}{\pi^{3}} \int_{0}^{\infty} d q_{0} n_{b}\left(q_{0}\right) \int_{q_{0}}^{2 \mu} d q q^{2} \frac{\operatorname{Im} \Pi_{T}^{(2)}}{q^{2}-q_{0}^{2}} \simeq \frac{g_{\mathrm{eff}}^{2} \mu^{2} T^{2}}{48 \pi^{2}}
\end{aligned}
$$

where $\Pi_{T}^{(2)} \equiv \Pi_{T}-\Pi_{T}^{\mathrm{HDL}}$ with $[33]$

$$
\operatorname{Im} \Pi_{T}^{(2)} \simeq-\frac{g_{\text {eff }}^{2} q q_{0}}{16 \pi}, \quad q \gg q_{0},
$$

while $\operatorname{Im} \Pi_{T}^{(2)} \rightarrow 0$ for $q \rightarrow q_{0}$. 
Similarly, in the longitudinal sector the non-HDL contribution to Eq. (64) is

$$
\begin{aligned}
& \frac{1}{N_{g}}\left[P_{L}^{\mathrm{II}, \mathrm{non}-\mathrm{HDL}}-\left.P_{L}^{\mathrm{II}, \mathrm{non}-\mathrm{HDL}}\right|_{T=0}\right] \\
& =-\frac{1}{2 \pi^{3}} \int_{0}^{\infty} d q_{0} n_{b}\left(q_{0}\right) \int_{q_{0}}^{2 \mu} d q q^{2} \frac{\operatorname{Im} \Pi_{L}^{(2)}}{q^{2}-q_{0}^{2}} \simeq \frac{g_{\mathrm{eff}}^{2} \mu^{2} T^{2}}{48 \pi^{2}}
\end{aligned}
$$

where

$$
\operatorname{Im} \Pi_{L}^{(2)} \simeq-\frac{g_{\text {eff }}^{2} q q_{0}}{8 \pi}, \quad q \gg q_{0}
$$

and again $\operatorname{Im} \Pi_{L}^{(2)} \rightarrow 0$ for $q \rightarrow q_{0}$.

The HDL part of the gluon self-energy, explicitly given by

$$
\begin{aligned}
& \Pi_{T}^{\mathrm{HDL}}\left(q_{0}, q\right)=m_{D}^{2} \frac{q_{0}^{2}}{2 q^{2}}\left(1+\frac{q_{0}^{2}-q^{2}}{2 q q_{0}} \log \frac{q_{0}-q}{q_{0}+q}\right), \\
& \Pi_{L}^{\mathrm{HDL}}\left(q_{0}, q\right)=m_{D}^{2} \frac{q^{2}-q_{0}^{2}}{q^{2}}\left(1+\frac{q_{0}}{2 q} \log \frac{q_{0}-q}{q_{0}+q}\right),
\end{aligned}
$$

with $m_{D}=g_{\text {eff }} \mu / \pi$ is the leading-order contribution at small $q_{0}, q \ll T$ provided $T \ll \mu$. In order to retain all contributions that are nonanalytic in $T$ at $T=0$, the HDL self-energies need to be kept unexpanded in

$$
\begin{aligned}
& \frac{1}{N_{g}}\left[P^{\mathrm{HDL}}-\left.P^{\mathrm{HDL}}\right|_{T=0}\right] \\
= & -\frac{1}{2 \pi^{3}} \int_{0}^{\infty} d q_{0} n_{b}\left(q_{0}\right) \int_{0}^{2 \mu} d q q^{2}\left[2 \operatorname{Im} \ln \left(q^{2}-q_{0}^{2}+\Pi_{T}^{\mathrm{HDL}}\right)+\operatorname{Im} \ln \left(\frac{q^{2}-q_{0}^{2}+\Pi_{L}^{\mathrm{HDL}}}{q^{2}-q_{0}^{2}}\right)\right]
\end{aligned}
$$

where we have dropped contributions from region III as being suppressed by explicit powers of $g_{\text {eff }}$.

Individually, the transverse and the longitudinal contributions depend logarithmically on the upper integration boundary, because

$$
\begin{aligned}
& \operatorname{Im} \ln \left(q^{2}-q_{0}^{2}+\Pi_{T}^{\mathrm{HDL}}\right) \rightarrow-\frac{g_{\mathrm{eff}}^{2} \mu^{2}}{4 \pi} \frac{q_{0}}{q^{3}} \\
& \operatorname{Im} \ln \left(\frac{q^{2}-q_{0}^{2}+\Pi_{L}^{\mathrm{HDL}}}{q^{2}-q_{0}^{2}}\right) \rightarrow+\frac{g_{\text {eff }}^{2} \mu^{2}}{2 \pi} \frac{q_{0}}{q^{3}}
\end{aligned}
$$

at large $q$. However, the combined expression (81) is saturated by soft momenta $q \ll \mu$, and the upper integration limit $2 \mu$ can be sent to infinity. This just amounts to dropping terms that are suppressed by explicit powers of $g_{\text {eff }}$.

The only other contribution that needs to be taken into account is $P_{\text {non- } n_{b}}$, which as discussed in Sect. IVC can be treated perturbatively to the order under consideration. Put together, the final result for $\Delta P=P-P^{0}$ is

$$
\begin{gathered}
\frac{1}{N_{g}}\left[\Delta P-\left.\Delta P\right|_{T=0}\right]=-\frac{g_{\text {eff }}^{2} \mu^{2} T^{2}}{48 \pi^{2}}-\frac{1}{2 \pi^{3}} \int_{0}^{\infty} d q_{0} n_{b}\left(q_{0}\right) \int_{0}^{\infty} d q q^{2}\left[2 \operatorname{Im} \ln \left(\frac{q^{2}-q_{0}^{2}+\Pi_{T}^{\mathrm{HDL}}}{q^{2}-q_{0}^{2}}\right)\right. \\
\left.+\operatorname{Im} \ln \left(\frac{q^{2}-q_{0}^{2}+\Pi_{L}^{\mathrm{HDL}}}{q^{2}-q_{0}^{2}}\right)\right]+O\left(g^{4} \mu^{2} T^{2}\right)
\end{gathered}
$$

where we have subtracted the ideal-gas pressure of the gluons by including a free propagator in the argument of the first logarithm. 
As indicated, this expression provides the leading terms in the temperature dependent part of the pressure and therefore the leading terms in entropy and specific heat. For $T \ll g_{\text {eff }} \mu$ the contribution to the pressure (as opposed to entropy and specific heat) is subleading compared to the three-loop result for the zero-temperature pressure obtained by Freedman and McLerran [37], Baluni [38], and Vuorinen [32]. However, when $T \gtrsim g_{\text {eff }} \mu$, its magnitude is comparable to $g_{\text {eff }}^{4} \mu^{4} \ln (c / g)$, the highest known term in the perturbative result of the $T=0$ pressure, and thus provides an extension of the result of Refs. [32, 37, 38] to non-zero temperatures in the domain $T \ll \mu$.

\section{B. HDL quasiparticle pole contribution}

In region I, i.e. $0 \leq q \leq q_{0}$, the HDL propagator has single poles for $q_{0} \geq \omega_{p}=m_{D} / \sqrt{3}$ at real $q_{0}=\omega_{T, L}(q)$, which allows us to carry out the $q_{0}$ integration, yielding

$$
\begin{aligned}
& \frac{1}{N_{g}}\left[P^{\mathrm{I}, \mathrm{HDL}}-\left.P^{\mathrm{I}, \mathrm{HDL}}\right|_{T=0}\right] \\
= & -\frac{T}{2 \pi^{2}} \int_{0}^{\infty} d q q^{2}\left[2 \ln \left(1-e^{-\omega_{T}(q) / T}\right)+\ln \left(\frac{1-e^{-\omega_{L}(q) / T}}{1-e^{-q / T}}\right)\right] .
\end{aligned}
$$

At small temperatures $T \ll \omega_{p}$, the contribution from transverse polarizations is suppressed by a factor $e^{-\omega_{p} / T}$ and thus does not show up at any finite order of a low-temperature expansion in terms of powers and logarithms of $T$. Using that for small $q \ll \omega_{p}$ the dispersion law of transverse gluons is given by [34]

$$
\omega_{T}(q)=\omega_{p}\left(1+\frac{3 q^{2}}{5 \omega_{p}^{2}}-O\left(q^{4} / \omega_{p}^{4}\right)\right)
$$

one can calculate the leading term as

$$
\frac{1}{N_{g}}\left[P_{T}^{\mathrm{I}, \mathrm{HDL}}-\left.P_{T}^{\mathrm{I}, \mathrm{HDL}}\right|_{T=0}\right] \simeq \frac{5}{12} \sqrt{\frac{5 \omega_{p}^{3} T^{5}}{3 \pi^{3}}} e^{-\omega_{p} / T}, \quad T \ll \omega_{p} .
$$

As discussed already in Sect. IVB, the low-temperature contribution of the longitudinal gluons involves a contribution $\propto T^{3}$ as well as exponentially suppressed terms. Using that

$$
\omega_{L}(q)=\omega_{p}\left(1+\frac{3 q^{2}}{10 \omega_{p}^{2}}-O\left(q^{4} / \omega_{p}^{4}\right)\right)
$$

one can show that

$$
\frac{1}{N_{g}}\left[P_{L}^{\mathrm{I}, \mathrm{HDL}}-\left.P_{L}^{\mathrm{I}, \mathrm{HDL}}\right|_{T=0}\right] \simeq-\frac{\pi^{2}}{90} T^{4}+\frac{5}{6} \sqrt{\frac{5 \omega_{p}^{3} T^{5}}{6 \pi^{3}}} e^{-\omega_{p} / T}, \quad T \ll \omega_{p} .
$$

At temperatures $\omega_{p} \ll T \ll \mu$ the dispersion relation of the longitudinal gluons approaches the light-cone exponentially, which gives an equally exponentially vanishing contribution to the pressure. The transverse gluons, on the other hand, tend to the mass hyperboloid $\omega_{T}(q) \rightarrow$ $\sqrt{q^{2}+m_{\infty}^{2}}$ with $m_{\infty}^{2}=m_{D}^{2} / 2$, yielding

$$
\frac{1}{N_{g}}\left[P^{\mathrm{I}, \mathrm{HDL}}-\left.P^{\mathrm{I}, \mathrm{HDL}}\right|_{T=0}\right] \simeq 2\left(\frac{\pi^{2}}{90} T^{4}-\frac{m_{\infty}^{2}}{24} T^{2}\right)=\frac{\pi^{2} T^{4}}{45}-\frac{g_{\mathrm{eff}}^{2} \mu^{2} T^{2}}{24 \pi^{2}}, \quad \omega_{p} \ll T \ll \mu .
$$




\section{HDL cut contribution}

For $q>q_{0}$, the HDL self-energies have an imaginary part $\propto m_{D}^{2}=g_{\text {eff }}^{2} \mu^{2} / \pi^{2}$ which corresponds to collisionless Landau damping of hard fermions. At low $q_{0} \sim T \ll g_{\text {eff }} \mu$, this provides the dynamical screening of quasistatic magnetic fields which is responsible for the anomalous terms in the low-temperature expansion of the specific heat, Eq. (54).

At higher temperatures $\omega_{p} \ll T \ll \mu$, it is instead electric Debye screening which gives the dominant contribution from soft momentum scales

$$
\begin{aligned}
\frac{1}{N_{g}}\left[P^{\mathrm{HDL}}-\left.P^{\mathrm{HDL}}\right|_{T=0}\right] \simeq & 2\left(\frac{\pi^{2}}{90} T^{4}-\frac{m_{\infty}^{2}}{24} T^{2}\right)+\frac{m_{D}^{3} T}{12 \pi} \\
& =\frac{\pi^{2} T^{4}}{45}-\frac{g_{\mathrm{eff}}^{2} \mu^{2} T^{2}}{24 \pi^{2}}+\frac{g_{\mathrm{eff}}^{3} \mu^{3} T}{12 \pi^{4}}, \quad \omega_{p} \ll T \ll \mu .
\end{aligned}
$$

(At still higher temperatures $T \gtrsim \mu$, one eventually has to replace the value of the HDL Debye mass by the hard-thermal-loop result [28]).

The appearance of a contribution $\propto m_{D}^{3}$ is traditionally referred to as plasmon effect. Our full HDL-resummed result (84) gives a unified description of this longitudinal plasmon effect with the anomalous (non-Fermi-liquid) contributions from transverse quasistatic fields which are only weakly screened, and interpolates between these two different effects. As the temperature is lowered, the longitudinal plasmon term $m_{D}^{3} T$ which is linear in $T$ gradually disappears and gets replaced by a quadratic term $\propto m_{D}^{2} T^{2} \ln \left(m_{D} / \mu\right)$, cf. Eq. (65). This then combines with the leading anomalous term $\propto m_{D}^{2} T^{2} \ln \left(T^{1 / 3} m_{D}^{2 / 3} / \mu\right)$ from the transverse sector, cf. Eq. (59) where $m_{D}$ enters through dynamical screening, and whose logarithmic dependence on the hard scale $\mu$ cancels that of the longitudinal sector.

\section{NUMERICAL RESULTS}

\section{A. Full HDL result versus low-temperature expansion}

We shall now turn to a numerical evaluation of entropy and specific heat following from the HDLresummed pressure (84). The corresponding expression for the entropy density is given explicitly by

$$
\begin{gathered}
\frac{1}{N_{g}}\left(\mathcal{S}-\mathcal{S}^{0}\right)=-\frac{g_{\mathrm{eff}}^{2} \mu^{2} T}{24 \pi^{2}}-\frac{1}{2 \pi^{3}} \int_{0}^{\infty} d q_{0} \frac{\partial n_{b}\left(q_{0}\right)}{\partial T} \int_{0}^{\infty} d q q^{2}\left[2 \operatorname{Im} \ln \left(\frac{q^{2}-q_{0}^{2}+\Pi_{T}^{\mathrm{HDL}}}{q^{2}-q_{0}^{2}}\right)\right. \\
\left.+\operatorname{Im} \ln \left(\frac{q^{2}-q_{0}^{2}+\Pi_{L}^{\mathrm{HDL}}}{q^{2}-q_{0}^{2}}\right)\right]+O\left(g_{\mathrm{eff}}^{4} \mu^{2} T\right)
\end{gathered}
$$

where $\mathcal{S}^{0}$ is the ideal-gas entropy density. Eq. (92) represents the leading interaction term at weak coupling for all $T \ll \mu$. It is essentially given by one universal function of the dimensionless variable $T /\left(g_{\text {eff }} \mu\right)$, which we define through

$$
\frac{8 \pi^{2}}{N_{g}\left(g_{\text {eff }} \mu\right)^{2} T}\left(\mathcal{S}-\mathcal{S}^{0}\right)=: \mathfrak{S}\left(\frac{T}{g_{\text {eff }}}\right)+O\left(g_{\text {eff }}^{2}\right),
$$

and which we have normalized such that the ordinary perturbative two-loop result [35] for the low-temperature entropy corresponds to $\mathfrak{S}=-1$.

In Fig. 2] we display the individual contributions to $\mathfrak{S}$ as provided by transverse and longitudinal quasiparticle poles (region I), and the Landau damping cut (region II). As one can see, the latter is 


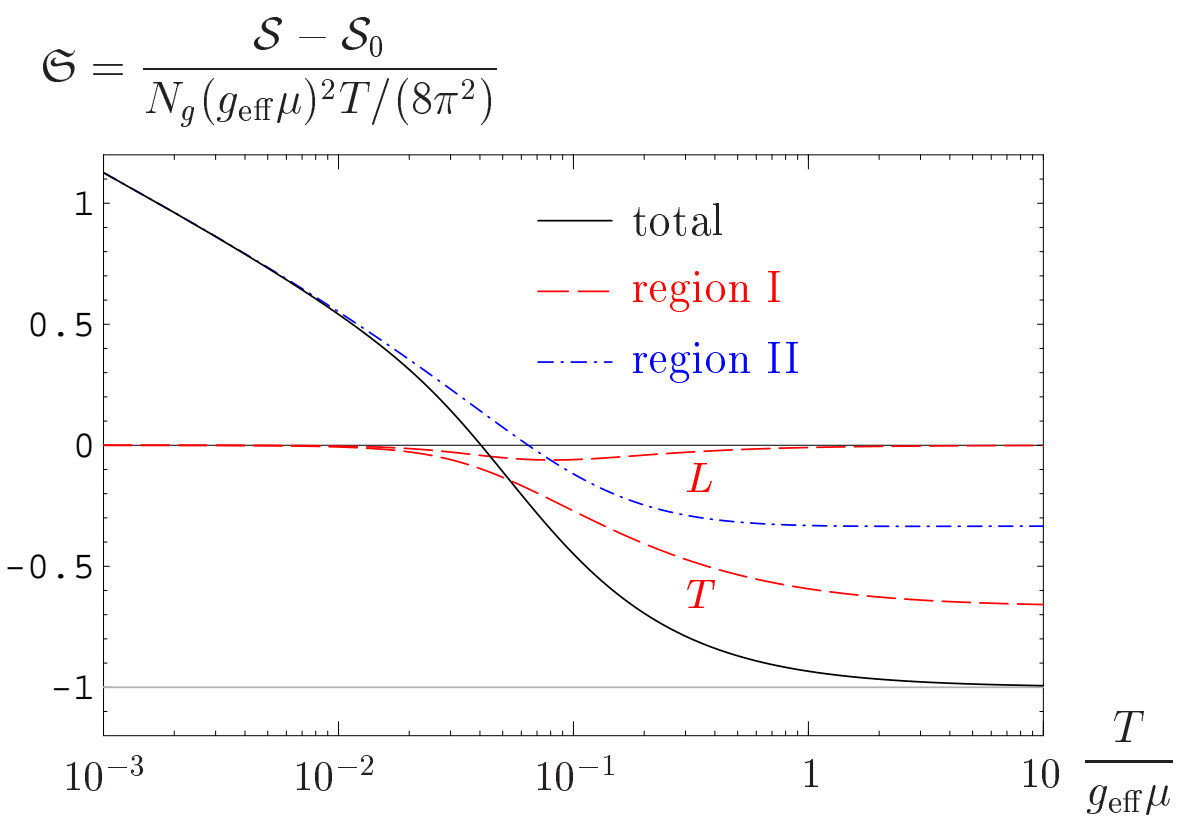

FIG. 2: The function $\mathfrak{S}\left(T /\left(g_{\text {eff }} \mu\right)\right)$ which determines the leading-order interaction contribution to the lowtemperature entropy. The normalization is such that $\mathfrak{S}=-1$ corresponds to the result of ordinary perturbation theory. The dash-dotted line shows the contribution from region II, comprising HDL Landau damping and hard contributions; the two dashed lines give the transverse (T) and longitudinal (L) quasiparticle pole contributions of region I.

responsible for the anomalous behavior of an interaction contribution which is positive for $T /(g \mu) \leq$ $0.0404 \ldots$ and is well reproduced by the low-temperature series involving logarithms and fractional powers of $T /\left(g_{\text {eff }} \mu\right)$. The quasiparticle contributions, on the other hand, behave as $T^{3}$ at small temperatures, but cannot be further expanded about $T=0$ due to terms involving $e^{-\omega_{p} / T}$.

We have in fact been able to perform the required numerical integrations with sufficient accuracy to explicitly check all the coefficients of the low-temperature expansion calculated in Sect. IV (and further ones up to order $T^{5} \ln T[36]$ ).

In Fig. 3 we compare the first few orders of the low-temperature series with the full HDL result. The low-temperature result up to and including the $T^{3} \ln T$ contribution to the entropy is a good approximation for $T /\left(g_{\text {eff }} \mu\right) \lesssim 0.04$ where the anomalous contributions dominate; for larger $T$ the non-expandable $e^{-\omega_{p} / T}$ terms in the quasiparticle pole contributions become important numerically.

\section{B. Comparison with nonperturbative large- $N_{f}$ results}

When applying our results to QED as well as QCD, the range of $T / \mu$ where one finds an excess of entropy and specific heat over the ideal-gas result will be the larger the higher the coupling is. However, we then have to expect more important perturbative corrections which are suppressed parametrically by further powers of $g^{2}$. In order to assess their importance, we compare with the special but exactly solvable case of infinite flavor number. This is done in Fig. 目 for the three values $g_{\text {eff }}\left(\bar{\mu}_{\mathrm{MS}}=2 \mu\right)=1,2,3$ where the heavy dots give the nonperturbative large- $N_{f}$ result of Ref. 16] and the full line represents the full HDL result (solid line, denoted by $\mathrm{HDL}^{+}$to remind of the inclusion of hard, perturbative terms). Also given is the low-temperature series up to and including the $T^{3} \ln T$ contributions.

While this certainly does not allow one to predict the reliability of our HDL result for real, 


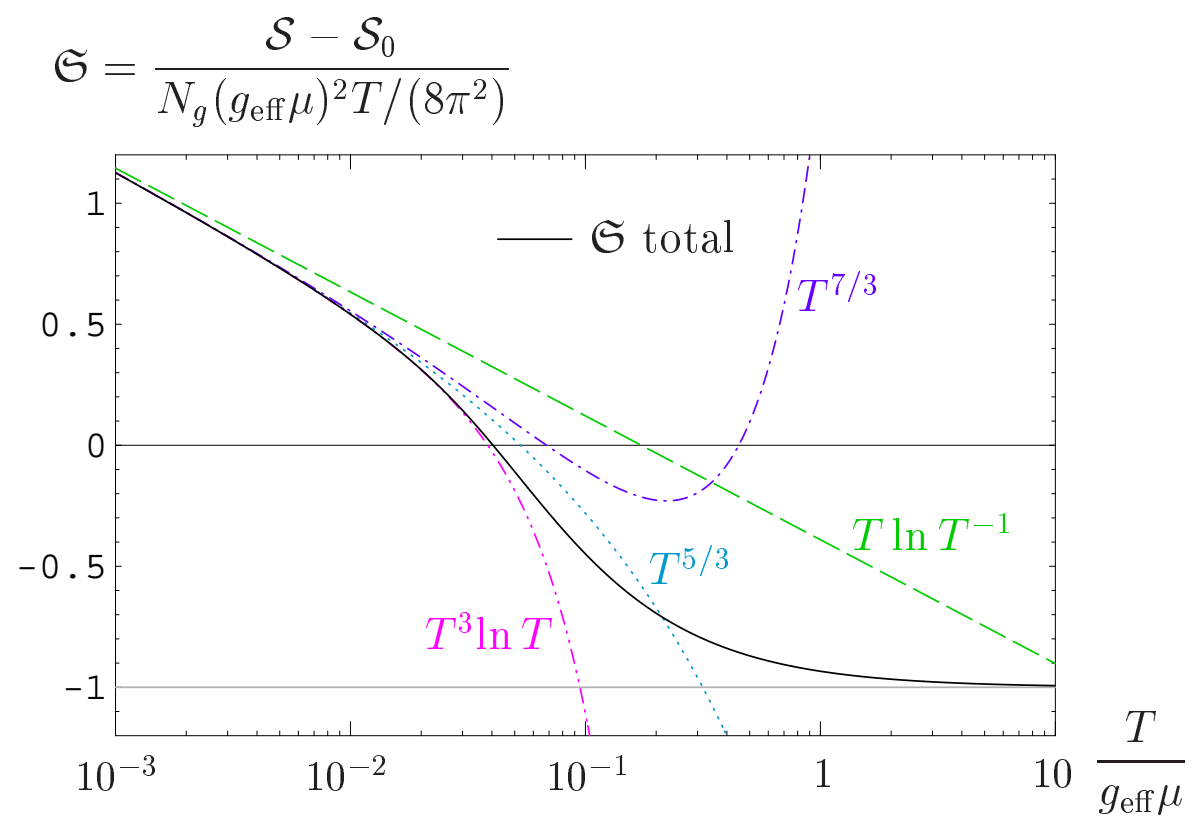

FIG. 3: The first few orders of the low-temperature series for the entropy as determined by Eq. (70) in comparison with the full HDL-resummed result.

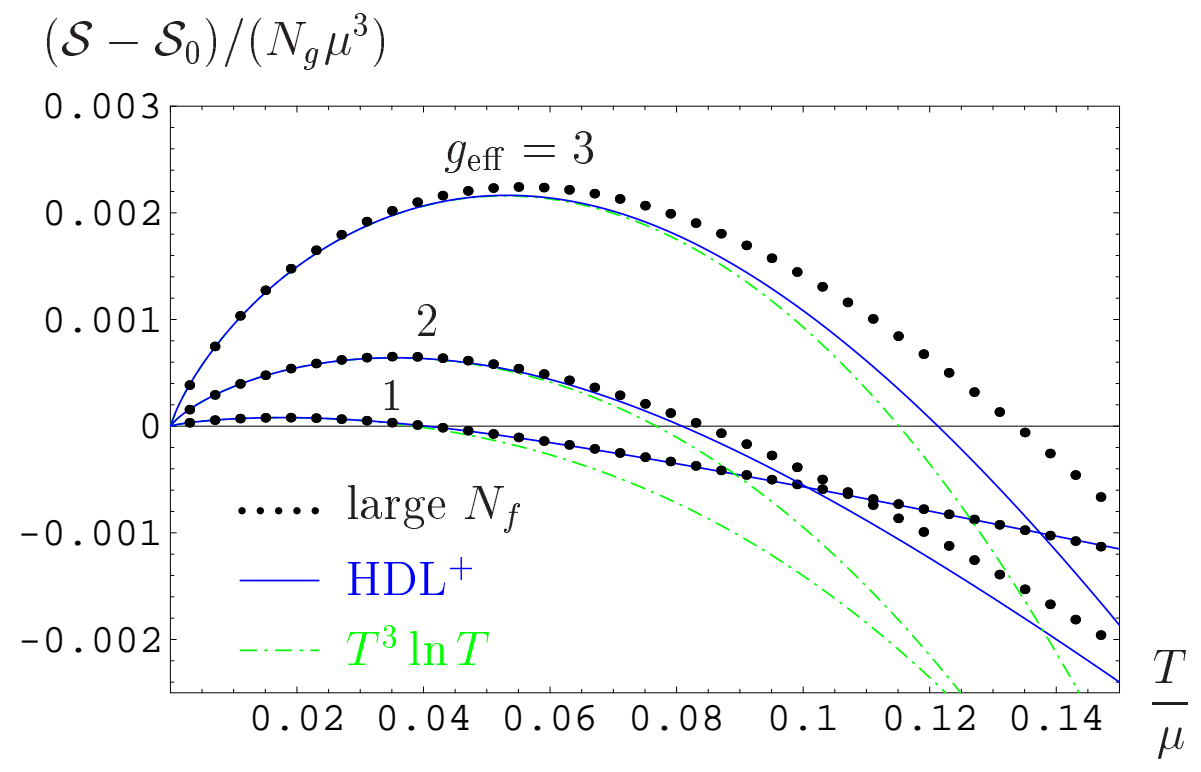

FIG. 4: Complete entropy density in the large- $N_{f}$ limit for the three values $g_{\text {eff }}\left(\bar{\mu}_{\mathrm{MS}}=2 \mu\right)=1,2,3$ (heavy dots), compared with the full HDL result (solid line) and the low-temperature series up to and including the $T^{3} \ln T$ contributions.

finite- $N_{f} \mathrm{QCD}$, it should give an idea of the errors to expect at least. Interestingly enough, in large- $N_{f}$ QCD the higher-order corrections seem to increase somewhat the range in $T / \mu$ where there is an excess of the entropy over its ideal-gas value.

By the same token, in Fig. [5 we display the renormalization scale dependence of the HDL result by varying the renormalization point by a factor of 2 around a central value of $\bar{\mu}_{\mathrm{MS}}=2 \mu$. 


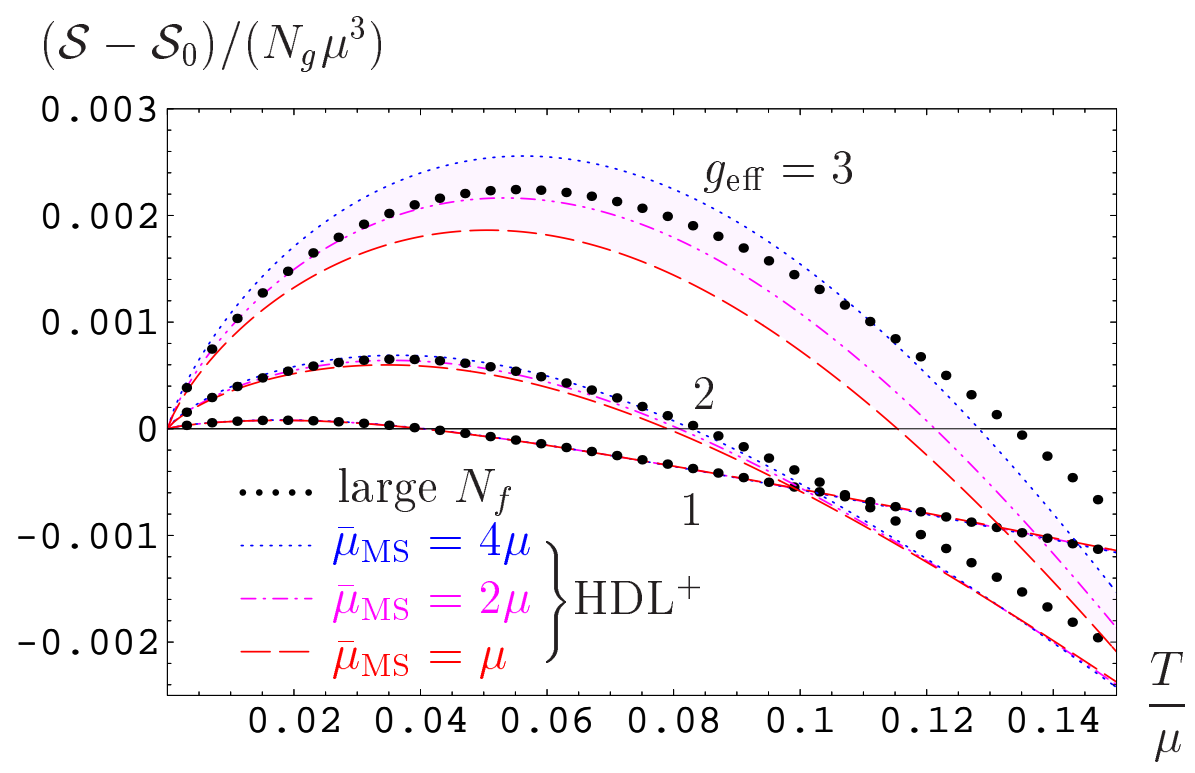

FIG. 5: Complete entropy density in the large- $N_{f}$ limit for the three values $g_{\text {eff }}\left(\bar{\mu}_{\text {MS }}=2 \mu\right)=1,2,3$ (heavy dots), compared with the HDL-resummed result when in the latter the renormalization scale is varied by a factor of 2 around $\bar{\mu}_{\mathrm{MS}}=2 \mu$.

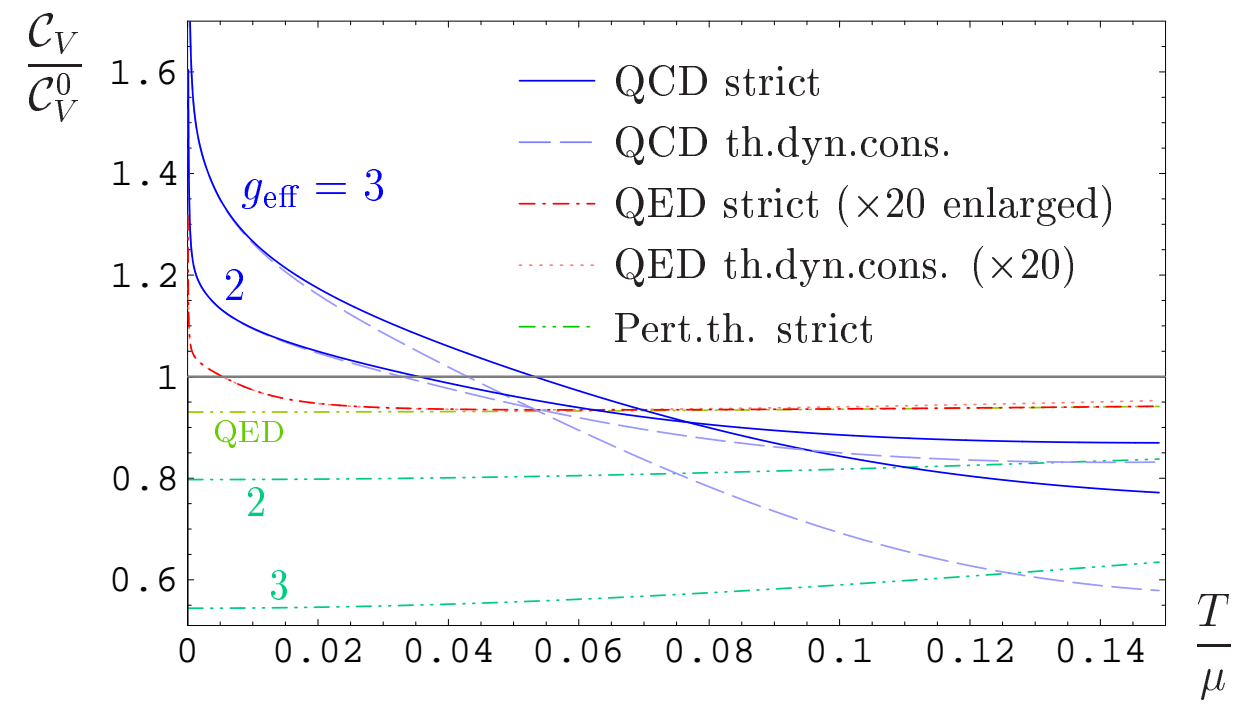

FIG. 6: The HDL-resummed result for the specific heat $\mathcal{C}_{v}$, normalized to the ideal-gas value for $g_{\text {eff }}=2$ and 3 corresponding to $\alpha_{s} \approx 0.32$ and 0.72 in two-flavor QCD, and $g_{\text {eff }} \approx 0.303$ for QED. The results labelled "strict" does not include anomalous contributions in the second term of Eq. (72) where the would be of higher order in $g_{\text {eff }}$, whereas "th.dyn.cons." refers to a less systematic but thermodynamically consistent evaluation. The deviation of the QED result from the ideal-gas value is enlarged by a factor of 20 to make it more visible.

\section{Specific heat}

In Fig. [6] we finally evaluate our result for the low-temperature specific heat at constant baryon density, $\mathcal{C}_{v}$. As can be seen from Eq. (172), this is a nonlinear functional of the thermodynamic potential. However, for $\mu \ll T$ and to leading order in $g_{\text {eff }}$ the anomalous contributions provided 
by Eq. (84) enter only through the logarithmic derivative of the entropy, and the nonlinear terms in Eq. (72) need only include the ideal-gas result. This defines the results in Fig. 6 labelled as "strict".

In order to have again an estimate of the uncertainties of undetermined higher-order contributions, we also computed $\mathcal{C}_{v}$ in a thermodynamically consistent manner directly from the pressure, given by Eq. (84) plus the perturbative zero-temperature result to order $g_{\mathrm{eff}}^{2}$. This has the slight deficiency of including higher-order terms in the second term of Eq. (172) beyond the accuracy of the first one. The "thermodynamically consistent" result is displayed in Fig. 6 by dashed lines, and one can see that there is not much difference in the region where $\mathcal{C}_{v} / \mathcal{C}_{v}^{0}$ is larger than one,

The results are given for three different couplings. The lines marked "QED" correspond to $g_{\text {eff }}=0.303$ or $\alpha_{Q E D} \approx 1 / 137$, and the results for $g_{\text {eff }}=2,3$ correspond to $\alpha_{s} \approx 0.32,0.72$ in two-flavor QCD. (Recall that $g_{\text {eff }}^{2} \equiv g^{2} N_{f} / 2$.) While in QED the effect is tiny (the deviations from the ideal-gas value have been enlarged by a factor of 20 in Fig. 6 to make them more visible), in QCD we find that there is an interesting range of $T / \mu$ where there is a significant excess of the specific heat over its ideal-gas value. whereas ordinary perturbation theory 35] would have resulted in a low-temperature limit of $\mathcal{C}_{v} / \mathcal{C}_{v}^{0}=1-2 \alpha_{s} / \pi$.

According to Ref. [12], the critical temperature for the color superconducting phase transition may be anywhere between 6 and $60 \mathrm{MeV}$, so with e.g. a quark chemical potential of $\mu=500 \mathrm{MeV}$ the range $T / \mu \geq 0.012$ in Fig. [6] might correspond to normal quark matter.

Thus, while the effect remains small in QED, it seems conceivable that the anomalous terms in the specific heat play a noticeable role in the thermodynamics of proto-neutron stars, in particular its cooling behavior in its earliest stages before entering color superconductivity [39, 40, 41].

If color superconductivity leaves some quark matter components unpaired, the larger values of $\mathcal{C}_{v} / \mathcal{C}_{v}^{0}$ at smaller $T / \mu$ may also be relevant for neutron stars with a quark matter core.

\section{SUMMARY}

For temperatures much smaller than the chemical potential of quarks (or electrons in the case of QED) we have computed the leading contribution to the interaction part of entropy and specific heat. For temperatures smaller than the Debye mass $\propto g_{\text {eff }} \mu$, the anomalous (non-Fermi-liquid) contributions become dominant. As we have discussed at length, this effect can be viewed either as a consequence of a logarithmic singularity of the fermion self-energy at the Fermi surface caused by long-range quasi-static magnetic interactions, or more directly as a contribution of the (imaginary part of the) transverse gauge boson propagator to the thermodynamic potential when the hard fermion degrees of freedom are integrated out first.

This latter approach proved to be advantageous for a systematic calculation beyond the wellknown leading-log approximation. We have obtained a hard-dense-loop resummed expression which continuously interpolates between the more familiar plasmon effect $\propto g^{3}$ coming from longitudinal Debye screened gauge bosons and the non-Fermi-liquid effects coming from only dynamically screened magnetic interactions. At temperatures much smaller than the Debye mass, we have obtained a low-temperature expansion starting with the well-known anomalous $T \ln T$ behaviour and involving also fractional powers of $T$ in subleading terms. The complete HDL-resummed result also contains contributions which do not show up at any finite order of the low-temperature expansion, being exponentially suppressed by factors of $e^{-\omega_{p} / T}$ at small $T$, but which become numerically important for intermediate temperatures.

Finally we have presented a numerical evaluation of our HDL-resummed result together with its low-temperature expansion, and we have compared with the exactly solvable large- $N_{f}$ limit of QCD and QED. This comparison seems to indicate that our leading-order-in- $g$ result, which is 
equally applicable to finite- $N_{f}$ QCD, is quite stable in the range of temperature where there is an excess of entropy and specific heat over their respective ideal-gas values. In QCD, where the coupling as well as the number of gauge bosons is much larger than in QED, the deviation from naive perturbation theory is appreciable for $T / \mu \lesssim 0.05$ and thus should be taken into account e.g. in studies of thermodynamic properties of quark matter in (proto-)neutron stars.

\section{Acknowledgments}

We gratefully acknowledge extensive discussions with Jean-Paul Blaizot and Urko Reinosa, and a correspondence with Thomas Schäfer. This work has been supported by the Austrian Science Foundation FWF, project no. 16387-N08 and the Austrian-French exchange program Amadée of the ÖAD, project no. $16 / 2003$.

\section{APPENDIX A: CALCULATION OF $\tilde{c}$}

From the terms which are explicitly shown in Eq. (58) we find the following contribution to the coefficient of $b^{12}$ in the pressure,

$$
\begin{aligned}
\tilde{c}_{T}^{(1)}= & \frac{1}{810 \pi^{2}}\left[-36 \pi^{2}+\right. \\
& \left.\left(\pi^{2}-8\right)\left(248-96 \gamma_{E}-9 \pi^{2}+48 \ln (4 \pi)+8640 \frac{\zeta^{\prime}(4)}{\pi^{4}}\right)\right] .
\end{aligned}
$$

However, some of the terms in the integrand of Eq. (58) that are formally of higher order than $b^{12}$ contribute also at the order of $b^{12}$, because the $x$-integration would be infrared divergent, were it not for the cutoff $x_{\min } \propto b^{6}$.

Since $x_{\text {min }}$ depends on $g_{\text {eff }}$ only through $b$, and since we can drop terms in the integrand of Eq. (58) involving $g_{\text {eff }}$ explicitly, it is sufficient to take the HDL self energy in the following. Then the gluon self energy can be written as

$$
\Pi_{T} \simeq \Pi_{T}^{\mathrm{HDL}}\left(\frac{q_{0}}{q}\right)=g_{\mathrm{eff}}^{2} \mu^{2} H_{T}\left(\frac{b^{2} y}{x^{1 / 3}}\right),
$$

with some function $H_{T}$. In the following we may neglect the explicit $q^{2}$ and $q_{0}^{2}$ in Eq. (53), because these two terms do not become singular for small $x .^{3}$ After expansion of the integrand with respect to $b$ we then obtain integrals of the type

$$
b^{6} \int_{x_{\text {min }}} d x\left(\frac{b^{2} y}{x^{1 / 3}}\right)^{n} \sim \frac{b^{12} y^{3}}{n-3} .
$$

Now we see clearly that from arbitrary powers of $b$ in the integrand we get contributions to the order $b^{12}$ in $P_{T}$. The case $n=3$ corresponds to the term of order $b^{12} \ln b$, which we have evaluated already in Sect. IVA For $n>3$ we can concentrate on the IR region and send to upper integration limit in Eq. (A3) to infinity. (The cases $n<3$ have been evaluated explicitly in Eq. (A1).) Furthermore we see from Eq. (A3) that from the $y$ integration we always get a factor

$$
\int_{0}^{\infty} \frac{d y y^{3}}{e^{y}-1}=\frac{\pi^{4}}{15}
$$

\footnotetext{
${ }^{3}$ However, $q^{2}$ and $q_{0}^{2}$ would have to be taken into account when summing up the IR contributions to the coefficient of $T^{6}$, since for this coefficient also less IR singular terms are important [36].
} 
The complete coefficient can thus be written as

$$
\tilde{c}_{T}=\tilde{c}_{T}^{(1)}-\frac{\pi^{4}}{15} \frac{1}{12 \pi^{4} b^{6}} \int_{4 \pi b^{6}}^{\infty} d x \sum_{n=8}^{\infty} \frac{b^{n}}{n !}\left(\left.\left[\frac{\partial^{n}}{\partial b^{n}} \arctan \left(\frac{\operatorname{Im} \Pi_{T}^{\mathrm{HDL}}}{\operatorname{Re} \Pi_{T}^{\mathrm{HDL}}}\right)\right]\right|_{b=0, y=1}\right) .
$$

This expression is in fact independent of $b$ (see Eq. (A3)). Therefore we may simply set $b=1$. Summing up the (Taylor) series, we find after the substitution $x=4 \pi z^{3}$

$$
\begin{aligned}
\tilde{c}_{T}= & \tilde{c}_{T}^{(1)}-\frac{\pi}{15} \int_{1}^{\infty} d z\left(\frac{128+3 \pi^{4} z^{3}-8 \pi^{2}\left(2+3 z^{2}\right)}{6 \pi^{3} z}\right. \\
& \left.+z^{2} \arctan \left[\frac{\pi\left(1-z^{2}\right)}{2 z+\left(z^{2}-1\right) \ln \left(\frac{z+1}{z-1}\right)}\right]\right) .
\end{aligned}
$$

From this expression we see that the complete HDL self energy is required for this coefficient (and not only the expansion for small $q_{0}$, which is sufficient for the fractional powers and the logarithmic terms). The remaining integral over the parameter $z$ can probably not be done analytically. Numerically one readily finds

$$
\tilde{c}_{T}=-0.00178674305 \ldots
$$

The constant $\tilde{c}_{L}^{\mathrm{II}}$ in Eq. (65) can be determined by summing up IR enhanced contributions in a completely analogous manner. The result is

$$
\begin{aligned}
\tilde{c}_{L}^{\mathrm{II}}= & \frac{1}{8640}\left(3 \pi^{4}-2 \pi^{2}\left(12-\pi^{2}\right)\left(-17+6 \gamma_{E}-6 \ln (\pi)-540 \frac{\zeta^{\prime}(4)}{\pi^{4}}\right)\right. \\
& -\frac{\pi}{30} \int_{1}^{\infty} d z\left(\frac{\pi\left(\pi^{2}+12\left(-1-z^{2}+z^{3}\right)\right)}{24 z}\right. \\
& \left.+z^{2} \arctan \left[-\frac{2 z}{\pi}+\frac{1}{\pi} \ln \left(\frac{z+1}{z-1}\right)\right]\right) \simeq 0.11902569216 \ldots
\end{aligned}
$$

[1] T. Holstein, R. E. Norton, and P. Pincus, Phys. Rev. B8, 2649 (1973).

[2] J. Gan and E. Wong, Phys. Rev. Lett. 71, 4226 (1993).

[3] S. Chakravarty, R. E. Norton, and O. F. Syljuåsen, Phys. Rev. Lett. 74, 1423 (1995).

[4] M. Y. Reizer, Phys. Rev. B40, 11571 (1989).

[5] C. M. Varma et al., Phys. Rev. Lett. 63, 1996 (1989).

[6] J. Polchinski, Effective field theory and the Fermi surface, hep-th/9210046.

[7] J. Polchinski, Nucl. Phys. B422, 617 (1994).

[8] C. Nayak and F. Wilczek, Nucl. Phys. B430, 534 (1994).

[9] D. T. Son, Phys. Rev. D59, 094019 (1999).

[10] W. E. Brown, J. T. Liu, and H.-c. Ren, Phys. Rev. D61, 114012 (2000); Phys. Rev. D62, 054013 (2000).

[11] Q. Wang and D. H. Rischke, Phys. Rev. D65, 054005 (2002).

[12] D. H. Rischke, Prog. Part. Nucl. Phys. 52, 197 (2004).

[13] D. Boyanovsky and H. J. de Vega, Phys. Rev. D63, 034016 (2001).

[14] D. Boyanovsky and H. J. de Vega, Phys. Rev. D63, 114028 (2001).

[15] G. D. Moore, JHEP 0210, 055 (2002); A. Ipp, G. D. Moore, and A. Rebhan, JHEP 0301, 037 (2003).

[16] A. Ipp and A. Rebhan, JHEP 0306, 032 (2003). 
[17] A. Ipp, A. Gerhold, and A. Rebhan, Phys. Rev. D69, 011901 (2004).

[18] L. D. Landau and E. M. Lifshitz, in Statistical Physics (Pergamon Press, London, 1958), Chap. 24, p. 71.

[19] J. M. Luttinger and J. C. Ward, Phys. Rev. 118, 1417 (1960).

[20] B. Vanderheyden and G. Baym, J. Stat. Phys. 93, 843 (1998).

[21] J. P. Blaizot, E. Iancu, and A. Rebhan, Phys. Rev. Lett. 83, 2906 (1999); Phys. Rev. D63, 065003 (2001).

[22] J. M. Luttinger and J. C. Ward, Phys. Rev. 118, 1417 (1960).

[23] C. Manuel, Phys. Rev. D62, 076009 (2000).

[24] E. Braaten and R. D. Pisarski, Nucl. Phys. B337, 569 (1990).

[25] T. Altherr and U. Kraemmer, Astropart. Phys. 1, 133 (1992).

[26] H. Vija and M. H. Thoma, Phys. Lett. B342, 212 (1995).

[27] C. Manuel, Phys. Rev. D53, 5866 (1996).

[28] M. Le Bellac, Thermal Field Theory (Cambridge University Press, Cambridge, UK, 1996).

[29] A. L. Fetter and J. D. Walecka, Quantum Theory of Many-Particle Systems (McGraw-Hill, New York, 1971).

[30] T. Schäfer and K. Schwenzer, Non-Fermi liquid effects in QCD at high density, hep-ph/0405053

[31] G. M. Carneiro and C. J. Pethick, Phys. Rev. B11, 1106 (1975).

[32] A. Vuorinen, Phys. Rev. D68, 054017 (2003).

[33] A. Ipp, Quantum corrections to thermodynamic properties in the large $N_{f}$ limit of the quark gluon plasma, doctoral thesis TU Vienna 2003, App. D, hep-ph/0405123

[34] H. A. Weldon, Phys. Rev. D26, 1394 (1982).

[35] J. I. Kapusta, Finite-temperature field theory (Cambridge University Press, Cambridge, UK, 1989).

[36] A. Gerhold, doctoral thesis TU Vienna, to appear.

[37] B. A. Freedman and L. D. McLerran, Phys. Rev. D16, 1147, 1169 (1977).

[38] V. Baluni, Phys. Rev. D17, 2092 (1978).

[39] N. Iwamoto, Phys. Rev. Lett. 44, 1637 (1980).

[40] G. W. Carter and S. Reddy, Phys. Rev. D62, 103002 (2000).

[41] K. W. Wong and M. C. Chu, Cooling of a new born compact star with QCD phase transition, astro-ph/0405601. 